\title{
Eigenvalue Problem for the 1-Laplace Operator
}

\section{Dissertation}

zur Erlangung des akademischen Grades

Doctor rerum naturalium

(Dr. rer. nat.)

vorgelegt

der Fakultät Mathematik und Naturwissenschaften

der Technischen Universität Dresden

von

Dipl.-Math. Zoja Milbers

geboren am 22.1.1981 in Kischinjow (Moldawien)

Gutachter: $\quad$ Prof. Friedemann Schuricht

Technische Universität Dresden

Prof. Bernd Kawohl

Universität zu Köln

Prof. Marco Degiovanni

Università Cattolica del Sacro Cuore, Brescia

Eingereicht am:

12.1.2009

Tag der Disputation: 23.3.2009 



\section{Contents}

$\begin{array}{ll}\text { Introduction } & 1\end{array}$

1 Eigenvalue Problem for the 1-Laplace Operator $\quad 7$

1.1 Eigenvalue Problem . . . . . . . . . . . . . . . . . . 7

1.2 Cheeger Sets. . . . . . . . . . . . . . . . . 15

1.3 Eigensolutions and Cheeger Sets . . . . . . . . . . . . . . . 19

2 Existence of a Sequence of Eigensolutions 21

2.1 Preliminary Considerations . . . . . . . . . . . . . . . . . . . 21

2.2 Tools of Nonsmooth Analysis . . . . . . . . . . . . . . . . 24

2.3 Higher Eigensolutions . . . . . . . . . . . . . . . . . 26

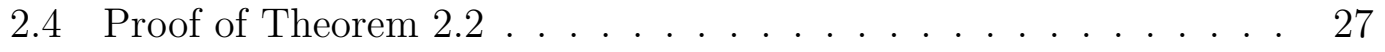

3 Necessary Condition for First Eigensolution by Inner Variations 43

3.1 Motivation and Formulation of the Necessary Condition . . . . . . 43

3.2 Proof of the Necessary Condition . . . . . . . . . . . . . . . . . . 47

$\begin{array}{lc}\text { Bibliography } & 69\end{array}$ 


\section{Introduction}

In the present work we consider the eigenvalue problem associated to the 1-Laplace operator, which formally has the form

$$
\Delta_{1} u:=\operatorname{Div}\left(\frac{D u}{|D u|}\right) .
$$

It has numerous applications, e. g. in mathematical image restoration, and therefore enjoys great interest of the scientific community. Moreover, the problem is also of purely theoretical interest, since it can be obtained from the well-known eigenvalue problem for the $p$-Laplace operator

$$
\Delta_{p} u:=\operatorname{div}\left(|D u|^{p-2} D u\right)
$$

by considering the limit $p \rightarrow 1$. The $p$-Laplace operator appears in many contexts in physics, for example in the study of non-Newtonian fluids, in nonlinear diffusion problems, in flows through porous media, and in plasma physics (cf. Diaz [18]).

Such problems are often studied by means of an associated variational problem. In the case $p \in(1, \infty)$ we consider the minimization of

$$
E_{p}(u):=\int_{\Omega}|D u|^{p} d x \rightarrow \operatorname{Min} !, \quad u \in W_{0}^{1, p}(\Omega)
$$

under the constraint

$$
G_{p}(u):=\int_{\Omega}|u|^{p} d x-1=0
$$

for a suitable domain $\Omega \subset \mathbb{R}^{n}$. The eigenvalue equation for the $p$-Laplace operator 
is the corresponding Euler-Lagrange equation given by

$$
-\operatorname{div}\left(|D u|^{p-2} D u\right)=\lambda|u|^{p-2} u \text { on } \Omega, \quad u=0 \text { on } \partial \Omega
$$

which can be obtained for a minimizer of this problem by classical methods of the calculus of variations. A lot of interesting results are known for this problem, such as the existence of a sequence of eigensolutions $\left(u_{k, p}, \lambda_{k, p}\right)_{k \in \mathbb{N}}$ and their $C_{\mathrm{loc}^{-}}^{1, \alpha}$ regularity. Moreover, the first eigenfunction is known to be positive (or negative) and unique up to scalar multiples. The eigenfunctions $u_{k, p} \in W_{0}^{1, p}(\Omega)$ are critical points of (1) under the constraint (2), while the corresponding eigenvalues $\lambda_{k, p} \rightarrow \infty$ as $k \rightarrow \infty$. This result can be obtained by means of the classical Ljusternik-Schnirelman theory for critical points.

However, the case $p=1$ confronts us with many difficulties. First of all it turns out that a more general space than $W_{0}^{1,1}(\Omega)$ is necessary in order to get existence of a minimizer. Moreover, the prescription of boundary values also has to be adapted, so that in the case $p=1$ it is reasonable to consider

$$
E(u):=\int_{\Omega} d|D u|+\int_{\partial \Omega}|u| d \mathcal{H}^{n-1} \rightarrow \operatorname{Min} !, \quad u \in B V(\Omega)
$$

under the constraint

$$
G(u):=\int_{\Omega}|u| d x-1=0 .
$$

It is known that a minimizer of this variational problem exists in $B V(\Omega)$, but in contrast to the case $p \in(1, \infty)$ it is not necessarily unique and it might change sign in $\Omega$. It has been shown by Fridman \& Kawohl in [24] that a suitable multiple of the characteristic function $u=\chi_{C}$ of the Cheeger set $C$ of $\Omega$ is a minimizer of (4) under the constraint (5), where, roughly speaking, a Cheeger set is a subset of $\Omega$ which minimizes the quotient $|\partial D| /|D|$ among all sets $D \subset \Omega$.

The derivation of an Euler-Lagrange equation for a minimizer of (4), (5) is a difficult task, since both $E$ and $G$ are nonsmooth functionals. The formal Euler-Lagrange equation obtained from (3) by taking $p \rightarrow 1$ contains expressions $D u /|D u|$ and $u /|u|$ which are not well defined, since characteristic functions are minimizers. A suitable substitute for this formal equation, obtained by a direct treatment of the variational problem (4), (5) with methods of nonsmooth analysis, has been deduced by Kawohl \& Schuricht in [31]. For any measurable 
selection $s$ of the set-valued sign function $\operatorname{Sgn}(u(x))$ there exists a vector field $z: \Omega \rightarrow B_{1}(0) \subset \mathbb{R}^{n}$, which depends on the choice of $s$, such that

$$
-\operatorname{Div} z(x)=\lambda s(x) \quad \text { on } \Omega \text {. }
$$

The vector field $z$ can be identified with $D u /|D u|$ if $|D u|$ is nonzero and is otherwise a suitable substitute for this expression. A measurable selection $s$ replaces $u /|u|$ by some value in $[-1,1]$ at points where $u$ vanishes. Hence, it turns out that for minimizers $u \in B V(\Omega)$ of (4), (5) infinitely many Euler-Lagrange equations have to be satisfied in general. We call this equation with many $s$ the multiple Euler-Lagrange equation and consider this problem to be the eigenvalue problem for the 1-Laplace operator.

In Chapter 1 we summarize some known results related to the $p$-Laplace operator, in particular in the case $p=1$. We give a survey on Cheeger sets and illuminate their connection to the eigenvalue problem for the 1-Laplace operator.

Higher eigensolutions of the eigenvalue problem related to the 1-Laplace operator are subject of Chapter 2. This question has not been considered so far, since it is not even clear how to define higher eigensolutions in our nonsmooth setting. On the one hand we might use the multiple Euler-Lagrange equation (6) for the definition, but it might be that this equation is satisfied only by the minimizer and thus it would be too restrictive for the definition of higher eigensolutions. By taking a closer look at the derivation of (6) we see that as a first condition we obtain equation (6) just for one measurable selection $s$. We call this equation with one $s$ the single Euler-Lagrange equation. But the single Euler-Lagrange equation also seems inappropriate for the definition of higher eigensolutions, since it is satisfied by "too many" functions as we demonstrate in the first section of Chapter 2. On the other hand it seems to be not possible to define them as critical points of (4) under the constraint (5), since $E$ and $G$ are not differentiable and $E$ is not even continuous. Nevertheless we choose this way and define them as critical points of $E$ under the constraint (5) by means of the weak slope, which is defined for continuous and even some classes of lower semicontinuous functions. Then we use the nonsmooth critical point theory developed by Degiovanni \& Marzocchi in [15] in order to obtain the existence of a sequence of critical points. Moreover, using a suitable nonsmooth version of the Lagrange multiplier theorem, 
we show that critical points have to satisfy the single Euler-Lagrange equation. The results of Chapter 2 can be found in Milbers \& Schuricht [34].

The situation is however not satisfactory, since it seems that not all of the functions satisfying the single Euler-Lagrange equation are critical points of (4), (5). One possibility to resolve this problem is to specify for which selections $s$ a single Euler-Lagrange equation has to be satisfied by higher eigensolutions. But this problem is completely open at the moment. Another possibility to diminish the number of possible solutions is the derivation of a different necessary condition for critical points of (4), (5). As a first step in this direction we deduce a further necessary condition for a minimizer of (4), (5) by means of inner variations in Chapter 3. There we consider variations of the domain $\Omega$ instead of those of a minimizer $u$ as in the derivation of the Euler-Lagrange equation. In the classical calculus of variations such an approach turns out to be useful if the minimizer is less regular than being in $C^{2}$, i. e. inner variations might produce an additional necessary condition which is different from the Euler-Lagrange equation. We adapt this method and use a suitable Lagrange multiplier rule which is applicable to our nonsmooth setting. It turns out that the new necessary condition is stronger than the single Euler-Lagrange equation, as we demonstrate on a model example. The derivation of a similar result for higher critical points of (4), (5) seems to be more complicated, but it appears to be a promising open challenge for the future. 


\section{Notation}

For a set $A$ we denote the boundary by $\partial A$, the closure by $\bar{A}$, and the interior by $\operatorname{int} A$. Its indicator function $I_{A}$ and its characteristic function $\chi_{A}$ are given by

$$
I_{A}(x):=\left\{\begin{array}{ll}
0 & \text { for } x \in A, \\
\infty & \text { otherwise }
\end{array} \quad \text { and } \quad \chi_{A}(x):= \begin{cases}1 & \text { for } x \in A \\
0 & \text { otherwise }\end{cases}\right.
$$

We denote by $|D|$ the volume of a set $D \subset \mathbb{R}^{n}$ and by $|\partial D|$ its perimeter. $B_{r}(u)$ stands for the open ball with center $u$ and radius $r$. We write Div $u$ for the divergence of a function $u$ in the distributional sense. The set-valued sign function on $\mathbb{R}$ is given by

$$
\operatorname{Sgn} \alpha:= \begin{cases}1 & \text { for } \alpha>0 \\ {[-1,1]} & \text { for } \alpha=0 \\ -1 & \text { for } \alpha<0\end{cases}
$$

The space of $q$-integrable functions on $\Omega$ is denoted by $L^{q}(\Omega)$ and its dual by $L^{q^{*}}(\Omega)$ where $\frac{1}{q}+\frac{1}{q^{*}}=1$. The Sobolev space $W^{1, p}(\Omega)$ consists of all $p$-integrable functions having $p$-integrable weak derivatives and functions in $W_{0}^{1, p}(\Omega)$ have zero trace in addition. The space $B V(\Omega)$ is the space of functions of bounded variation and $|D u|$ is the total variation measure for these functions. For a Radon measure $\mu$ we denote by $\mu\lfloor A$ its restriction to a set $A$ and by $f \mu$ its product with a $\mu$-summable function $f$. The $k$-dimensional Hausdorff measure is denoted by $\mathcal{H}^{k}$. For a Banach space $X$ its dual is $X^{*}$ and $\langle\cdot, \cdot\rangle$ is the duality form on $X \times X^{*}$. We write $\partial F(u)$ both for the subdifferential of a convex function $F$ and for Clarke's generalized gradient of a locally Lipschitz continuous function $F$. By $\delta F(u, v)$ we denote the directional derivative and by $F^{0}(u, v)$ Clarke's generalized directional derivative of $F$ at $u$ in direction $v$. For a matrix $M \in \mathbb{R}^{n \times n}$ we denote by adj $M$ its adjoint, defined for a regular matrix as $M^{-1} \operatorname{det} M$, and by $\operatorname{tr}(M)$ its trace. By id $\in \mathbb{R}^{n \times n}$ we mean the identity matrix. By $\delta_{j k}$ we denote the Kronecker delta. 


\section{Acknowledgments}

In the first place I wish to express my deep and sincere gratitude to my supervisor, Prof. Dr. Friedemann Schuricht who was abundantly helpful and offered invaluable assistance, support and guidance. I also wish to thank Prof. Dr. Marco Degiovanni for stimulating discussions and brilliant ideas, and especially for inviting me to Brescia. I am grateful to Prof. Dr. Bernd Kawohl for his generosity and his nondecreasing interest in my work.

I would like to thank my colleagues in Cologne who were always eager to discuss my mathematical problems with me, especially Dr. Daniel Habeck, Kerstin Does, Nicole Schadewaldt, and Enea Parini. I am also grateful to my colleagues in Dresden who gave me the feeling of being at home at work, especially to the members of the "Kaffeerunde".

At last, I owe my most sincere gratitude to my husband Max Milbers who has always encouraged me to continue my work, to our baby for being so patient with me, and to the rest of my family for their constant support. 


\section{Chapter 1}

\section{Eigenvalue Problem for the 1-Laplace Operator}

In this chapter we recall some known results concerning the eigenvalue problem for the $p$-Laplace operator, while we emphasize the singular case $p=1$. We define the notion of a Cheeger set and state its basic properties. At last we illuminate the connection between Cheeger sets and eigensolutions of the 1-Laplace operator.

\subsection{Eigenvalue Problem}

Let us consider the minimization problem

$$
\begin{gathered}
E_{p}(u):=\int_{\Omega}|D u|^{p} d x \rightarrow \operatorname{Min} !, \quad u \in W_{0}^{1, p}(\Omega), \\
G_{p}(u):=\int_{\Omega}|u|^{p} d x-1=0,
\end{gathered}
$$

for $p \in(1, \infty)$ where $\Omega \subset \mathbb{R}^{n}$ is a bounded domain. For most of the results following no regularity assumptions are needed for the boundary $\partial \Omega$. The corresponding Euler-Lagrange equation

$$
-\operatorname{div}\left(|D u|^{p-2} D u\right)=\lambda|u|^{p-2} u \text { on } \Omega, \quad u=0 \text { on } \partial \Omega,
$$


which is called the eigenvalue problem for the p-Laplace operator, can be derived from (1.1), (1.2) by means of the classical Lagrange multiplier rule, while we interpret the equation in the weak sense. We say that $u \in W_{0}^{1, p}(\Omega), u \neq 0$ is an eigenfunction and $\lambda \in \mathbb{R}$ a corresponding eigenvalue of the $p$-Laplace operator, if they satisfy equation (1.3).

This problem has been studied extensively in the literature during the past decades and many interesting results have been obtained. We give a short survey on the results related to the eigenvalue problem (1.3), parts of which can be found in $[32]$.

The existence of a positive (or negative) eigenfunction can be established by minimizing the Rayleigh quotient

$$
\frac{E_{p}(v)}{G_{p}(v)+1}
$$

over all $v \in W_{0}^{1, p}(\Omega)$ with $v \neq 0$. Using a normalized minimizing sequence $\left(\varphi_{k}\right)_{k \in \mathbb{N}} \subset C_{0}^{\infty}(\Omega)$ we obtain a function $u \in W_{0}^{1, p}(\Omega)$ such that

$$
\frac{E_{p}(u)}{G_{p}(u)+1}=\min _{0 \neq v \in W_{0}^{1, p}(\Omega)} \frac{E_{p}(v)}{G_{p}(v)+1} .
$$

The compactness argument needed in the existence proof is provided by the Rellich-Kondrachov Theorem. Then the classical Lagrange multiplier rule shows that $u$ is a solution of (1.3). If $u$ is minimizing, so is $|u|$ and therefore also $|u|$ satisfies the equation. Since $|u| \geq 0$, we must have $|u|>0$ by Harnack's inequality. By continuity either $u>0$ or $u<0$ in $\Omega$, cf. Lindqvist [32].

The following uniqueness result for the first eigenfunction is due to Kawohl \& Lindqvist [30, Theorem 1.1].

Theorem 1.1. Let $\Omega \subset \mathbb{R}^{n}$ be a bounded domain. Then any positive solution of (1.3) is unique up to scalar multiplication.

This function is called the first eigenfunction of the $p$-Laplace operator and the corresponding $\lambda_{1, p}$ is the first eigenvalue, i. e. the smallest eigenvalue, of the 
$p$-Laplace operator. The first eigenvalue $\lambda_{1, p}$ can also be characterized by means of the Rayleigh quotient

$$
\lambda_{1, p}=\min _{0 \neq v \in W_{0}^{1, p}(\Omega)} \frac{E_{p}(v)}{G_{p}(v)+1},
$$

cf. e.g. [30]. Moreover, if $\lambda>\lambda_{1, p}$, there are no positive eigenfunctions with eigenvalue $\lambda$, cf. [30, Lemma 3.1] and the restriction of a higher eigenfunction to a nodal domain, which is a maximal connected open subset of $\{x \in \Omega \mid u(x) \neq 0\}$, is a first eigenfunction there.

However, surprisingly little is known about higher eigenvalues and eigenfunctions in the case $p \neq 2$. In the case $p=2$ the Laplace operator has a discrete spectrum of eigenvalues $\lambda_{1}<\lambda_{2} \leq \lambda_{3} \leq \ldots$ and $\lambda_{k} \rightarrow \infty$ as $k \rightarrow \infty$. For $p \neq 2$ the eigenfunctions can be characterized as critical points of $E_{p}$ as defined in (1.1) under the constraint (1.2) by means of the Ljusternik-Schnirelman theory (see Rabinowitz [37] for the general theory). There we consider symmetric, i.e. if $v \in A$ then also $-v \in A$, closed subsets $A$ of a Banach space and define the Kransnoselskij genus of $A$ as the smallest $k \in \mathbb{N}$ for which there exists a continuous odd function $\psi: A \rightarrow \mathbb{R}^{k} \backslash\{0\}$. In our setting we denote by $\mathcal{A}_{k}$ the collection of all closed symmetric sets $A \subset W_{0}^{1, p}(\Omega)$ such that the Krasnoselskij genus of $A$ is at least $k$ and the set $\left\{v \in A \mid\|v\|_{p}=1\right\}$ is compact. Then higher eigenvalues are characterized by

$$
\lambda_{k, p}=\inf _{A \in \mathcal{A}_{k}} \sup _{v \in A} \frac{E_{p}(v)}{G_{p}(v)+1} .
$$

García Azorero \& Peral Alonso have proved in [25] that there exists a nondecreasing sequence $\left(\lambda_{k, p}\right)_{k \in \mathbb{N}}$ of nonnegative eigenvalues and eigenfunctions $\left(u_{k, p}\right)_{k \in \mathbb{N}}$ of the $p$-Laplace operator with $\lambda_{k, p} \rightarrow \infty$ as $k \rightarrow \infty$. For an eigenfunction $u_{k, p}$ it is known that it has at most $(2 k-2)$ nodal domains if $\Omega \subset \mathbb{R}^{n}$ is a bounded domain with smooth boundary, cf. Drábek \& Robinson [20, Theorem 3.3]. However, for $p \neq 2$ it is not known whether this sequence contains all eigenvalues and many questions remain unanswered regarding the properties of the spectrum beyond $\lambda_{2, p}$. In contrast to the Laplace operator, the nonlinear spectrum has not been proved to be discrete, not even when the domain $\Omega$ is a ball.

The following result, which is due to Anane \& Tsouli [3], shows that the method at least gives the correct $\lambda_{1, p}$ and $\lambda_{2, p}$ and the second eigenvalue is well defined 
and characterized by (1.5).

Theorem 1.2. Let $\Omega$ be a bounded domain and $\lambda_{2, p}$ as defined in (1.5). Then

$$
\begin{aligned}
\lambda_{2, p} & =\min \left\{\lambda_{p} \mid \lambda_{p} \text { is an eigenvalue with sign changing eigenfunction }\right\} \\
& =\min \left\{\lambda_{p} \mid \lambda_{p} \text { is an eigenvalue and } \lambda_{p}>\lambda_{1, p}\right\} .
\end{aligned}
$$

Thus, between $\lambda_{1, p}$ and $\lambda_{2, p}$, which are always distinct, there are no eigenvalues. Unfortunately, it has not even been proved that it is impossible that every real number $\lambda>\lambda_{2, p}$ is an eigenvalue. Neither is anything known about the multiplicity of the higher eigenvalues.

A useful result concerning the regularity of eigenfunctions of the $p$-Laplace operator has been shown by DiBenedetto in [19, Theorem 2].

Theorem 1.3. Let $\Omega \subset \mathbb{R}^{n}$ be open, $u \in W^{1, p}(\Omega)$ a solution of (1.3). Then $u \in C_{\text {loc }}^{1, \alpha}(\Omega)$ where $\alpha \in(0,1)$ depends only on $n$ and $p$.

A consequence of this result is that in regular domains the boundary condition is satisfied in the classical sense, i. e. as a pointwise condition. For example, any domain satisfying an exterior cone condition is regular enough in the case $p \leq n$, cf. Gariepy \& Ziemer [26]. Moreover, if $p>n$, then every boundary point is regular.

Let us now consider the highly singular case $p=1$. In this case we have to minimize the functional $\int_{\Omega}|D u| d x$ under the side constraint $\int_{\Omega}|u| d x=1$, which are both functionals with linear growth. Minimizers of such functionals typically belong to the space $B V(\Omega)$ of functions of bounded variation on $\Omega$, and not necessarily to $W^{1,1}(\Omega)$. Therefore it is natural to study the minimization problem in $B V(\Omega)$ for $p=1$. But since the trace operator on $B V(\Omega)$ has merely weak continuity properties, the usual prescription of the trace is too restrictive if we work in $B V(\Omega)$. Thus it seems to be reasonable to consider the variational problem

$$
E(u):=\int_{\Omega} d|D u|+\int_{\partial \Omega}|u| d \mathcal{H}^{n-1} \rightarrow \operatorname{Min} !, \quad u \in B V(\Omega)
$$




$$
G(u):=\int_{\Omega}|u| d x-1=0
$$

in the case $p=1$, where the surface integral replaces the Dirichlet data in a generalized way, cf. Kawohl \& Schuricht [31, p. 518].

Let $\Omega \subset \mathbb{R}^{n}$ be a bounded domain with Lipschitz boundary for the rest of this section.

The existence of a minimizer of (1.6), (1.7) has been stated, e. g., by Kawohl \& Schuricht in [31, Theorem 3.2].

Theorem 1.4. Problem (1.6), (1.7) has a solution $u \in B V(\Omega)$.

However, in contrast to the case $p \in(1, \infty)$ the solution does not have to be unique as we will see below.

The formal limit of (1.3) for $p \rightarrow 1$ is the eigenvalue problem

$$
-\operatorname{Div}\left(\frac{D u}{|D u|}\right)=\lambda \frac{u}{|u|} .
$$

This equation is not well defined if we have in mind that typical minimizers of (1.6), (1.7) are piecewise constant and even vanish on a set of positive measure. In order to give meaning to problem (1.8), variational problem (1.6), (1.7) has to be studied more carefully. Here the functional to be minimized contains the nonsmooth 1-homogeneous total variation and the $L^{1}$-norm is prescribed as a nonsmooth constraint. The lack of differentiability of $E$ and $G$ requires more general tools than usually used in the calculus of variations.

One way to approach this problem is to approximate the degenerate problem by a regular one and to study the limit. This has been done by Fridman \& Kawohl in [24] and by Demengel in [17]. The drawback of such an approximation is that the result might depend on the special smoothing.

The straightforward derivation of a necessary condition for minimizers of (1.6), (1.7) has been done by Kawohl \& Schuricht in [31]. Since $E$ and $G$ are convex, a necessary condition for a minimizer $u$ should employ the convex subdifferentials $\partial E(u), \partial G(u)$. But if we want to characterize these subdifferentials, which are subsets of the dual space $B V(\Omega)^{*}$ we are confronted with the difficulty that not 
much is known about the structure of the space $B V(\Omega)^{*}$, cf. Ambrosio et al. [2]. It turns out that the structure of the elements in $\partial E(u), \partial G(u)$ can be derived if $B V(\Omega)$ is considered as a subspace of $L^{q}(\Omega)$ with $q>1$ and if $E$ and $G$ are extended on $L^{q}(\Omega)$ by

$$
E(u):= \begin{cases}\int_{\Omega} d|D u|+\int_{\partial \Omega}|u| d \mathcal{H}^{n-1} & \text { for } u \in B V(\Omega), \\ \infty & \text { for } u \in L^{q}(\Omega) \backslash B V(\Omega)\end{cases}
$$

and

$$
G(u):=\int_{\Omega}|u| d x-1=0 \quad \text { for } u \in L^{q}(\Omega) .
$$

Note that this has been done in [31] only for the case $\frac{n}{n-1} \leq q<\infty$. However, all of the corresponding results in [31] can be extended to the general case $1<q<\infty$ (cf. also Andreu-Vaillo et al. [4]). The following characterization of subdifferentials of $E$ and $G$ can be found in Kawohl \& Schuricht [31, Proposition $4.23]$.

Proposition 1.5. The functional $E$ according to (1.9) is convex and lower semicontinuous on $L^{q}(\Omega)$. Moreover, $u_{E}^{*} \in \partial E(u)$ for $u \in L^{q}(\Omega)$ if and only if there exists a vector field $z \in L^{\infty}\left(\Omega, \mathbb{R}^{n}\right)$ with

$$
\begin{aligned}
\|z\|_{L^{\infty}} \leq 1, \quad u_{E}^{*} & =-\operatorname{Div} z \in L^{q^{*}}(\Omega), \\
E(u) & =\left\langle u_{E}^{*}, u\right\rangle=-\int_{\Omega} u \operatorname{Div} z d x .
\end{aligned}
$$

If $E(u)>0$, then $\|z\|_{L^{\infty}}=1$.

Proposition 1.6. The functional $G$ according to (1.10) is convex and Lipschitz continuous on $L^{q}(\Omega)$. Moreover, we have $u_{G}^{*} \in \partial G(u)$ for $u \in L^{q}(\Omega)$ if and only if

$$
u_{G}^{*}(x) \in \operatorname{Sgn}(u(x)) \quad \text { a.e. on } \Omega \text {. }
$$

Note that this result has been shown in [31] for $u \mapsto \int_{\Omega}|u| d x$ but, obviously, it is also valid for our $G$ which differs merely by a constant.

The following theorem of Kawohl \& Schuricht [31, Corollary 4.18] provides a suitable substitute for (1.8) (cf. also Demengel [17] for a partial result). 
Theorem 1.7. Let $u \in B V(\Omega)$ be a minimizer of (1.6), (1.7). Then for each measurable selection $s(x) \in \operatorname{Sgn}(u(x))$ a.e. on $\Omega$ there exists a corresponding vector field $z \in L^{\infty}\left(\Omega, \mathbb{R}^{n}\right)$ satisfying

$$
\begin{gathered}
\|z\|_{L^{\infty}}=1, \quad \operatorname{Div} z \in L^{n}(\Omega), \\
E(u)=-\int_{\Omega} u \operatorname{Div} z d x
\end{gathered}
$$

such that

$$
-\operatorname{Div} z=\lambda s \quad \text { a.e. on } \Omega, \quad \lambda=E(u)
$$

We call (1.13) combined with the coupling conditions (1.11), (1.12) relating $z$ to $u$ the eigenvalue problem for the 1-Laplace operator. A solution $u$ is said to be an eigenfunction according to the eigenvalue $\lambda$.

Let us take a closer look at Theorem 1.7. As a first necessary condition for a minimizer $u$ of (1.6), (1.7) we obtain that there is one sign function $s$ and a corresponding vector field $z$ such that (1.13) is satisfied with $\lambda=E(u)$. This result can be obtained by applying a suitable Lagrange multiplier rule to the variational problem (1.6), (1.7). We call this necessary condition with one $s$ the single Euler-Lagrange equation. But the particular form of the problem allows special arguments, which exploit the relation between certain cones and imply many equations as necessary condition for a minimizer $u$. We call such a necessary condition with many $s$ the multiple Euler-Lagrange equation. Since $u$ is known to be zero on large sets, as we will see below, we get infinitely many equations for $u$ in general, which is a surprising and remarkable result.

A useful equivalent formulation of the coupling condition (1.12) has been stated by Milbers \& Schuricht in [34].

Proposition 1.8. Let $u \in B V(\Omega)$ and let $z \in L^{\infty}\left(\Omega, \mathbb{R}^{n}\right)$ satisfy $\operatorname{Div} z \in L^{n}(\Omega)$ and $\|z\|_{L^{\infty}} \leq 1$. Then (1.12) is equivalent to the two conditions

$$
-[z, \nu](x) \in \operatorname{Sgn}(u(x)) \quad \mathcal{H}^{n-1} \text {-a.e. on } \partial \Omega
$$

and

$$
D u=z|D u|
$$


in the sense of measures on $\Omega$.

It is an interesting question, to what extent the coupling conditions combined with a fixed $s$ determine the vector field $z$. Since it seems that $|z(x)|<1$ at least on some ball $B \subset \Omega$ for a typical solution $z$, we clearly can add any divergence free vector field with sufficiently small amplitude and support on $B$ in order to get a further solution $z$. This way we always obtain infinitely many vector fields satisfying the necessary condition for a fixed selection $s$. However, a much more interesting question is how much freedom we have for $z$ on the boundary $\partial \Omega$ where, in general, the coupling conditions do not completely prescribe $z$. This question has been investigated in [34] for the special case of a square $\Omega \subset \mathbb{R}^{2}$. It turns out that for each measurable selection $s$ there exist even infinitely many vector fields $z$, differing on a part of the boundary $\partial \Omega$, which satisfy (1.13) on $\Omega$, with $s$ and $z$ related to $u$ as in Theorem 1.7.

Now the question for higher eigensolutions for the case $p=1$ is natural but confronts us with fundamental difficulties. The first problem that we have to face is the proper definition of higher eigensolutions. It seems that the single Euler-Lagrange equation with one $s$ is too weak for a definition of eigensolutions, since it is satisfied by "very many" functions as we will show shortly. On the other hand, there is some analytical evidence that the multiple Euler-Lagrange equation, taking into account any $s$, might be true merely for a minimizer $u$ of (1.6), (1.7) and, hence, it would be too strong for a definition of eigensolutions. Thus we cannot decide for which functions $s$ equation (1.13) should be satisfied for a higher eigenfunction $u$. Consequently, we do not have a precise replacement for the formal eigenvalue problem (1.8) as basis for a definition of higher eigensolutions. Recall that in the case $p \in(1, \infty)$ eigenfunctions $u$ are critical points of $E_{p}$ subject to the constraint $G_{p}(u)=0$. However, we cannot define critical points in the usual way in the limit case $p=1$, since $E$ and $G$ are not differentiable. We will take a closer look at these problems in Chapter 2. 


\subsection{Cheeger Sets}

We define the Cheeger constant of a nonempty open bounded set $\Omega \subset \mathbb{R}^{n}$ by

$$
h(\Omega):=\inf _{D \subset \Omega} \frac{|\partial D|}{|D|}
$$

with $D$ varying over all nonempty sets $D \subset \Omega$ of finite perimeter, cf. Alter \& Caselles [1]. Originally the Cheeger constant has been defined by Cheeger in [10] in a slightly different manner. We call the expression

$$
Q(D):=|\partial D| /|D|
$$

the Cheeger quotient of $D$ and a set $C \subset \Omega$ is called a Cheeger set of $\Omega$ if $Q(C)=h(\Omega)$. Note that the minimum in (1.16) cannot be obtained at a set $D$ whose distance from the boundary of $\Omega$ is positive, otherwise we could diminish the Cheeger quotient $Q(D)$ by rescaling $D$ with a factor larger than one. Note that $C$ is a Cheeger set of $\Omega$ if and only if it minimizes

$$
\min _{D \subset \Omega}(|\partial D|-Q(C)|D|) .
$$

We call a set $\Omega \subset \mathbb{R}^{n}$ calibrable if $\Omega$ minimizes the problem

$$
\min _{D \subset \Omega}(|\partial D|-Q(\Omega)|D|),
$$

or, equivalently, if $\Omega$ is a Cheeger set of itself. If $C$ is a Cheeger set of $\Omega$, then $C$ is always calibrable. A simple example of a calibrable set is a ball, where the Cheeger constant can be calculated explicitly. If we consider $B_{r}(0) \subset \mathbb{R}^{n}$, then its Cheeger constant is

$$
h\left(B_{r}(0)\right)=\frac{\left|\partial B_{r}(0)\right|}{\left|B_{r}(0)\right|}=\frac{n}{r} .
$$

Before we illuminate the relation between Cheeger sets and the eigenvalue problem for the 1-Laplace operator, let us give a short survey on the general properties of Cheeger sets. First of all we address the question of uniqueness.

In the general case the Cheeger set is not necessarily unique, as Figure 1.1 shows. 


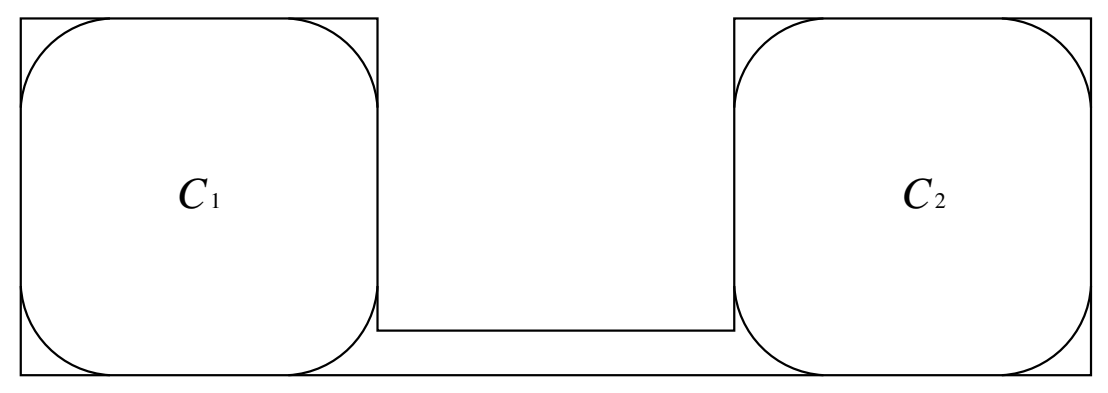

Figure 1.1: Cheeger sets of a barbell-type domain

In this case the "rounded squares" $C_{1}, C_{2}$ and the set $C_{1} \cup C_{2}$ are all Cheeger sets. Moreover, there might even exist a continuous family of Cheeger sets. One example for this case is the L-shaped planar domain, as has been shown by Parini in [36]. Here we obtain infinitely many Cheeger sets by shifting the half-circle on

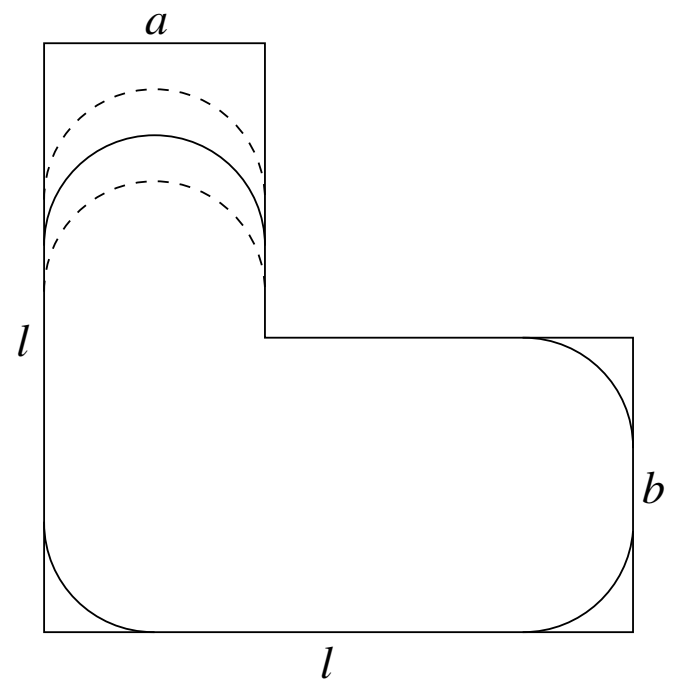

Figure 1.2: Cheeger sets of an L-shaped domain

the top-left side upwards or downwards (for some special ratio of $a, b, l$ ).

The uniqueness of a Cheeger set has been known for a long time in the case $n=2$ for a convex domain $\Omega$. A much more general uniqueness result for convex $\Omega \subset \mathbb{R}^{n}$ has recently been proved by Alter \& Caselles in [1, Theorem 1$]$.

Theorem 1.9. Every open convex bounded set $\Omega \subset \mathbb{R}^{n}$ has a unique Cheeger set. The Cheeger set is convex and its boundary is of class $C^{1,1}$. 
Moreover, uniqueness can be obtained by small perturbations of the set $\Omega$, cf. Caselles et al. [9, Theorem 1].

Theorem 1.10. Let $\Omega \subset \mathbb{R}^{n}$ be an open set with finite volume. Then, for any compact set $K \subset \Omega$ there exists a bounded open set $\Omega_{K} \subset \Omega$ such that $K \subset \Omega_{K}$ and $\Omega_{K}$ has a unique Cheeger set.

A consequence of this result is, that given any open set with finite volume, for all $\varepsilon>0$, we can find a set $\Omega_{\varepsilon} \subset \Omega$ such that $\left|\Omega \backslash \Omega_{\varepsilon}\right|<\varepsilon$ and $\Omega_{\varepsilon}$ has a unique Cheeger set.

The following result concerning regularity of the boundary of Cheeger sets has been proved in [9, Theorem 2].

Theorem 1.11. Let $\Omega$ be a bounded open set with boundary of class $C^{1,1}$. Then any Cheeger set $C$ of $\Omega$ has boundary of class $C^{1,1}$, except on a closed singular subset of $\partial \Omega$ of dimension at most $(n-8)$.

Moreover, we have a useful result describing the geometry of a Cheeger set, cf. Gonzalez et al. [28, Theorem 2] (see also Stredulinsky \& Ziemer [39]).

Theorem 1.12. Let $\Omega \subset \mathbb{R}^{n}$ be an open bounded set with Lipschitz boundary and $D \subset \Omega$ a set minimizing perimeter among all sets with prescribed volume. Let $x_{1}, x_{2} \in \partial D \cap \Omega$ be regular points and $f_{1}, f_{2}$ analytic functions describing $\partial D$ locally near $x_{1}, x_{2}$. Then

$$
\operatorname{div}\left(\frac{D f_{1}}{\sqrt{1+\left|D f_{1}\right|^{2}}}\right)=\operatorname{div}\left(\frac{D f_{2}}{\sqrt{1+\left|D f_{2}\right|^{2}}}\right)
$$

Since the Cheeger set $C$ minimizes perimeter among all sets $D \subset \Omega$ with $|C|=$ $|D|$, the theorem says that the surface $\partial C \cap \Omega$ has constant mean curvature. It can even be shown that the mean curvature equals $h(\Omega)$ in the regular points of $\partial C \cap \Omega$, cf. Fridman \& Kawohl [24]. In the case of $\Omega \subset \mathbb{R}^{2}$ this readily implies that $\partial C \cap \Omega$ consists of circular arcs with radius $r=1 / h(\Omega)$. If in addition $\Omega$ is convex, then the union of discs of radius $r=1 / h(\Omega)$ inside $\Omega$ uniquely defines its Cheeger set. But note that for nonconvex planar domains this construction method might fail, cf. Kawohl \& Lachand-Robert [29, p. 13]. 
Example 1.13. If $\Omega$ is a square given by $(0, a) \times(0, a)$, then the Cheeger set can be represented by a union of discs with radius $r=1 / h(\Omega)$ in $\mathbb{R}^{2}$, see Figure 1.3. The Cheeger constant is then the minimum of

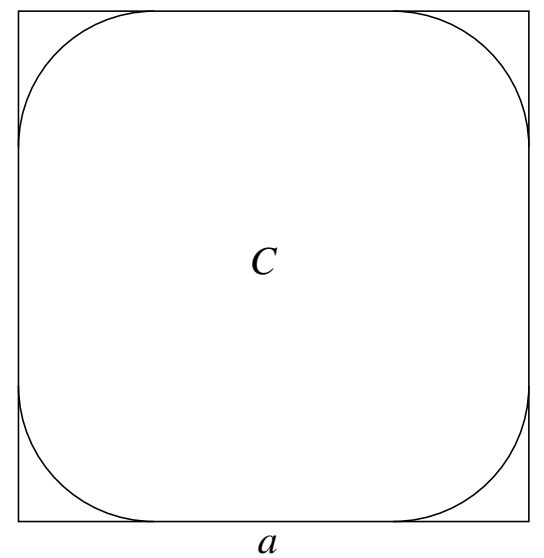

Figure 1.3: Cheeger set of a square

$$
\frac{|\partial C|}{|C|}=\frac{4(a-2 r)+2 \pi r}{a^{2}-4\left(r^{2}-\frac{1}{4} \pi r^{2}\right)}
$$

over $r$. We calculate the minimum of this expression and get

$$
r=\frac{2-\sqrt{\pi}}{4-\pi} a
$$

and thus

$$
h(\Omega)=\frac{4-\pi}{(2-\sqrt{\pi}) a},
$$

cf. $[24$, p. 4].

However, finding a Cheeger set of a given set $\Omega$ is usually a difficult task and only some particular cases seem to be explicitly known. For example a disk or an annulus are known to be calibrable. Kawohl \& Lachand-Robert have recently given a constructive method for the computation of the Cheeger set of any planar convex polygon in [29]. 


\subsection{Eigensolutions and Cheeger Sets}

Now we want to illustrate what Cheeger sets and eigensolutions of the $p$-Laplace operator have in common.

First of all, the Cheeger constant gives us a lower bound for the first eigenvalue of the $p$-Laplace operator. Cheeger has proved the following statement for the special case $p=2$ in $[10]$ and the extension of it to a general $p \in(1, \infty)$ has been done by Matei in [33].

Theorem 1.14. Let $\Omega \subset \mathbb{R}^{n}$ be a bounded simply connected domain with suffciently smooth boundary. Then the first eigenvalue of the p-Laplace operator can be estimated from below by

$$
\lambda_{1, p} \geq\left(\frac{h(\Omega)}{p}\right)^{p} .
$$

Fridman \& Kawohl have treated the case $p \rightarrow 1$ in [24, Corollary 6] and obtained the following result.

Theorem 1.15. Let $\Omega \subset \mathbb{R}^{n}$ be a bounded simply connected domain with suffciently smooth boundary. For the first eigenvalue $\lambda_{1,1}$ of the 1-Laplace operator we have

$$
\lambda_{1,1}=h(\Omega) .
$$

The following result relating Cheeger sets of a given domain $\Omega$ to a first eigenfunction of the 1-Laplace operator has also been proved in [24, Theorem 8].

Theorem 1.16. Let $\Omega \subset \mathbb{R}^{n}$ be a bounded simply connected domain with suffciently smooth boundary. If $C \subset \Omega$ is a Cheeger set of $\Omega$, then $u=\alpha \chi_{C} \in B V(\Omega)$, $\alpha \in \mathbb{R} \backslash\{0\}$, is a first eigenfunction of the 1-Laplace operator. If $v \in B V(\Omega)$ is a first eigenfunction of the 1-Laplace operator, then almost all of its level sets $\{x \in \Omega|| v(x) \mid>t\}$ are Cheeger sets of $\Omega$.

Let us discuss the significance of this result. First of all we obtain a result on the existence of a Cheeger set of $\Omega$. We know that a minimizer $u \in B V(\Omega)$ of (1.6), (1.7) exists in any bounded domain $\Omega$ with Lipschitz boundary by Theorem 1.4. 
Since it satisfies the constraint $G(u)=0$ it must be nonzero and therefore nontrivial level sets exist. Now, by Theorem 1.16, almost all of these level sets are Cheeger sets.

Moreover, if we know that a Cheeger set $C$ of $\Omega$ exists, then $u=\frac{1}{|C|} \chi_{C}$ is a first eigenfunction by Theorem 1.16. If we consider the analytical problem

$$
\inf _{0 \neq v \in B V(\Omega)} \frac{E(v)}{G(v)+1},
$$

which is equivalent to (1.6), (1.7), then a minimizer $u$ must satisfy

$$
\lambda_{1,1}=\frac{E(u)}{G(u)+1},
$$

cf. also equation (1.4) for $p \in(1, \infty)$. But then the Rayleigh quotient in (1.19) becomes $|\partial C| /|C|$, which is equal to the Cheeger constant $h(\Omega)$ by definition. Since $u$ satisfies $G(u)=0$, we thus get the identity

$$
\lambda=E(u)=h(\Omega),
$$

cf. also (1.13), for the first eigenvalue $\lambda=\lambda_{1,1}$ and a corresponding eigenfunction $u$ of the 1-Laplace operator.

Furthermore, now we can address the question of whether or not a first eigenfunction can be unique in the case $p=1$. Recall that the first eigenfunction of the $p$-Laplace operator with $p \in(1, \infty)$ is unique up to scalar multiplication and positive (or negative) in $\Omega$. In the case $p=1$ both these properties are lost. We see that uniqueness of the first eigenfunction fails if the Cheeger set of $\Omega$ is not unique, such as in the case of barbell-type or L-shaped domains, see Figures 1.1 or 1.2. For instance for the barbell-type domain any function $u(x)=c_{1} \chi_{C_{1}}(x)+c_{2} \chi_{C_{2}}(x), c_{1}, c_{2} \in \mathbb{R}$, with $\left|c_{1}\right|\left|C_{1}\right|+\left|c_{2}\right|\left|C_{2}\right|=1$ is a first eigensolution. This example also shows that a first eigenfunction can have a sign change in $\Omega$. Even if we have an eigenfunction which does not change sign in $\Omega$, it will not be positive in general, since it may have large nullsets.

Of course a regularity result similar to Theorem 1.3 also cannot be expected in the case $p=1$, since characteristic functions are first eigenfunctions, i. e. not even continuous in the general case. 


\section{Chapter 2}

\section{Existence of a Sequence of Eigensolutions}

The question of higher eigensolutions for the 1-Laplace operator is the subject of this chapter. We define eigenfunctions of the 1-Laplace operator as critical points of (1.6), (1.7) by means of the weak slope. Then we verify the existence of a sequence $\left(u_{k}\right)_{k \in \mathbb{N}}$ of critical points by means of nonsmooth critical point theory and show that each such eigenfunction $u_{k}$ has to satisfy a single Euler-Lagrange equation.

\subsection{Preliminary Considerations}

We have already mentioned that we do not have a precise replacement for the formal eigenvalue problem (1.8) as basis for the definition of higher eigensolutions. For a first eigensolution we use the multiple Euler-Lagrange equation as definition, see Theorem 1.7. But this seems to be too restrictive for the definition of higher eigensolutions, since we expect that merely a minimizer of the variational problem satisfies the multiple equation. Below we demonstrate that also the single EulerLagrange equation is inappropriate for the definition, since it allows too many solutions, providing a continuum of eigenvalues. 
Let $B=B_{r}\left(x_{0}\right) \subset \Omega$ be a ball with radius $r$ and center $x_{0}$ such that $\partial B$ does not touch $\partial \Omega$, cf. Figure 2.1. We show that the $B V(\Omega)$-function

$$
v(x):=\frac{1}{|B|} \chi_{B}(x)
$$

satisfies the single Euler-Lagrange equation for one $s$ and a corresponding $\lambda$. We construct the associated vector field $z$ in $B$ and $\Omega \backslash B$ separately. Inside $B$

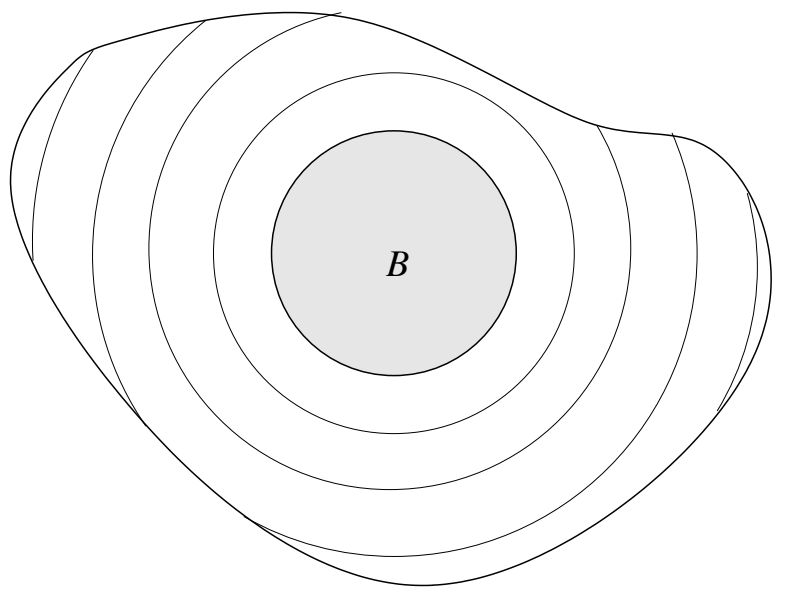

Figure 2.1: Foliation of $\Omega \backslash B$

the function $v$ is positive and thus we have to find a corresponding vector field $z \in L^{\infty}\left(\Omega, \mathbb{R}^{n}\right)$ satisfying the coupling conditions (1.11), (1.12) and

$$
-\operatorname{Div} z=\lambda \quad \text { a.e. on } B
$$

with

$$
\lambda=E(u)=\frac{|\partial B|}{|B|}=\frac{n}{r} .
$$

If we set

$$
z(x)=\frac{x_{0}-x}{r} \text { on } B
$$

then

$$
-\operatorname{div} z(x)=\frac{n}{r} .
$$

Thus $z$ satisfies $-\operatorname{div} z=\lambda$ and the coupling conditions on $B$. Note that for 
$x \in \partial B$ we have $z(x)=\frac{x_{0}-x}{\left|x_{0}-x\right|}$ since $\left|x_{0}-x\right|=r$ there. We set

$$
z(x)=\frac{x_{0}-x}{\left|x_{0}-x\right|} \quad \text { on } \Omega \backslash B,
$$

i. e. $z$ is the unit normal vector field to the foliation of $\Omega \backslash B$ obtained by concentric spheres and pointing to the center $x_{0}$, cf. Figure 2.1. In this case we have

$$
-\operatorname{div} z(x)=\frac{n-1}{\left|x_{0}-x\right|} .
$$

Thus we get a continuous vector field

$$
z(x):= \begin{cases}\frac{x_{0}-x}{r} & \text { for } x \in B, \\ \frac{x_{0}-x}{\left|x_{0}-x\right|} & \text { for } x \in \Omega \backslash B,\end{cases}
$$

which satisfies (1.11), (1.12). Note that $\operatorname{Div} z \in L^{n}(\Omega)$ and it jumps across $\partial B$ from $n / r$ to $(n-1) / r$. We set

$$
s(x):= \begin{cases}1 & \text { for } x \in B, \\ \frac{n-1}{n} \frac{r}{\left|x_{0}-x\right|} & \text { for } x \in \Omega \backslash B .\end{cases}
$$

Clearly, $s(x) \in[-1,1]$, i. e. $s$ is a measurable selection of $\operatorname{Sgn}(v(x))$ and

$$
-\operatorname{Div} z=\frac{n}{r} s(x) \quad \text { on } \Omega
$$

is satisfied.

Notice that there is a continuum of eigenvalues $\lambda \in\left(n / r_{0}, \infty\right)$, where $r_{0}$ denotes the radius of the largest ball contained in $\Omega$. Hence, we have constructed infinitely many solutions of the single Euler-Lagrange equation, since the radius $r$ of $B$ is allowed to vary between 0 and $r_{0}$. However, such functions $v$ cannot be minimizers of (1.6), (1.7) since a ball $B$ which does not touch $\partial \Omega$ cannot minimize the Cheeger quotient. 


\subsection{Tools of Nonsmooth Analysis}

The notion of weak slope has been introduced by Degiovanni \& Marzocchi in [15]. Let $X$ be a metric space endowed with metric $m$ and let $E: X \rightarrow \mathbb{R}$ be a continuous function. For every $u \in X$ we denote by $|d E|(u)$ the supremum of all $\sigma \in[0, \infty)$ for which there exist $\delta>0$ and a continuous map

$$
\mathcal{H}: B_{\delta}(u) \times[0, \delta] \rightarrow X
$$

such that for all $v \in B_{\delta}(u)$ and all $t \in[0, \delta]$

$$
\begin{gathered}
m(\mathcal{H}(v, t), v) \leq t \\
E(\mathcal{H}(v, t)) \leq E(v)-\sigma t
\end{gathered}
$$

The extended real number $|d E|(u)$ is called the weak slope of $E$ at $u$. Note that for differentiable functions the weak slope corresponds to the norm of the gradient. Now we consider a lower semicontinuous function $E: X \rightarrow \mathbb{R} \cup\{\infty\}$. We define the domain of $E$ by

$$
\mathcal{D}(E):=\{u \in X \mid E(u)<\infty\}
$$

and the epigraph of $E$ by

$$
\operatorname{epi}(E):=\{(u, \xi) \in X \times \mathbb{R} \mid E(u) \leq \xi\} \text {. }
$$

The set $X \times \mathbb{R}$ will be endowed with the metric

$$
m((u, \xi),(v, \mu))=\sqrt{m(u, v)^{2}+(\xi-\mu)^{2}}
$$

and epi $(E)$ with the induced metric. Using the continuous function

$$
\mathscr{G}_{E}: \operatorname{epi}(E) \rightarrow \mathbb{R}, \quad \mathscr{G}_{E}(u, \xi)=\xi
$$

we define the weak slope of $E$ at $u \in \mathcal{D}(E)$ as

$$
|d E|(u):= \begin{cases}\frac{\left|d \mathscr{G}_{E}\right|(u, E(u))}{\sqrt{1-\left|d \mathscr{G}_{E}\right|(u, E(u))^{2}}} & \text { for }|d \mathscr{G}|(u, E(u))<1 \\ \infty & \text { for }|d \mathscr{G}|(u, E(u))=1\end{cases}
$$


When $E$ is finite and continuous on $X$ this definition is consistent with the definition of the weak slope for continuous functions. Occasionally we denote the weak slope of $E$ at $u$ by $|d E|^{X}(u)$ in order to indicate that it is taken in the metric space $X$. The idea of this definition is to reduce the study of the lower semicontinuous function $E$ to that of the Lipschitz continuous function $\mathscr{G}_{E}$.

We say that $u \in \mathcal{D}(E)$ is a critical point of $E$ if $|d E|(u)=0$. The value $c \in \mathbb{R}$ is called a critical value of $E$ if there exists a critical point $u \in \mathcal{D}(E)$ of $E$ with $E(u)=c$. Note that if $(u, E(u)) \in \operatorname{epi}(E)$ is a critical point of $\mathscr{G}_{E}$ then $u$ is also a critical point of $E$. The bijective correspondence between the critical points of $E$ and those of $\mathscr{G}_{E}$ is given if

$$
\inf \left\{\left|d \mathscr{G}_{E}\right|(u, \xi) \mid E(u)<\xi\right\}>0
$$

cf. Canino \& Degiovanni [7, Theorem 1.5.5]. If $E$ is finite and continuous, we have $\left|d \mathscr{G}_{E}\right|(u, \xi)=1$ whenever $E(u)<\xi$. The same property holds for some important classes of lower semicontinuous functions (cf. Canino \& Perri [8], Corvellec et al. [14], Degiovanni \& Marzocchi [15]).

We are interested in critical points of $E$ under a constraint $G(u)=0$ where $G: X \rightarrow \mathbb{R}$ is a locally Lipschitz continuous function. We set

$$
K:=\{u \in X \mid G(u)=0\}
$$

and call $u \in \mathcal{D}(E)$ a critical point of $E$ with respect to $K$ if $u$ is a critical point of $E$ on the metric space $K$ with induced metric $m$ of $X$.

The following result gives us a useful characterization of critical points of $E$ with respect to $K$.

Lemma 2.1. Let $E: X \rightarrow \mathbb{R} \cup\{\infty\}$ be a lower semicontinuous function and let $u \in \mathcal{D}(E) \cap K$. Then $|d E|^{K}(u)=0$ if and only if $\left|d\left(E+I_{K}\right)\right|^{X}(u)=0$.

Proof. For the calculation of the weak slope of $E+I_{K}$ on $X$ and of $E$ with respect to $K$, we must turn to functions

$$
\mathscr{G}_{E+I_{K}}: \operatorname{epi}\left(E+I_{K}\right) \rightarrow \mathbb{R} \quad \text { and } \quad \mathscr{G}_{E}: \operatorname{epi}(E) \cap(K \times \mathbb{R}) \rightarrow \mathbb{R}
$$


respectively, as defined in (2.5). First we observe that

$$
\begin{aligned}
\operatorname{epi}\left(E+I_{K}\right) & =\left\{(v, \mu) \in X \times \mathbb{R} \mid\left(E+I_{K}\right)(v) \leq \mu\right\} \\
& =\{(v, \mu) \in K \times \mathbb{R} \mid E(v) \leq \mu\} \\
& =\operatorname{epi}(E) \cap(K \times \mathbb{R}) .
\end{aligned}
$$

Thus $\mathscr{G}_{E+I_{K}}$ and $\mathscr{G}_{E}$ are defined on the same metric space, since the metric $m$ as defined in (2.4) is the same in both cases. By definition (2.5) we have

$$
\mathscr{G}_{E+I_{K}} \equiv \mathscr{G}_{E}
$$

Hence,

$$
\left|d \mathscr{G}_{E+I_{K}}\right|\left(u,\left(E+I_{K}\right)(u)\right)=\left|d \mathscr{G}_{E}\right|(u, E(u))
$$

for each $u \in \mathcal{D}(E) \cap K$. Therefore

$$
\left|d\left(E+I_{K}\right)\right|^{X}(u)=|d E|^{K}(u) \quad \text { for all } u \in \mathcal{D}(E) \cap K
$$

which implies the assertion.

\subsection{Higher Eigensolutions}

We intend to verify the existence of a sequence of pairs $\left(u_{k},-u_{k}\right)_{k \in \mathbb{N}}$ of critical points of (1.6), (1.7) and derive an Euler-Lagrange equation as necessary condition for critical points.

Since we need the subdifferentials of $E$ and $G$ for the derivation of the necessary condition, we extend them trivially to the space $L^{q}(\Omega)$ with $q<\frac{n}{n-1}$ as already demonstrated in (1.9), (1.10). This special choice of $q$ makes sure that $B V(\Omega)$ is compactly embedded into $L^{q}(\Omega)$, which will be crucial for the proof of the Palais-Smale condition. Based on this setting we say that $E$ as defined in (1.6) has a critical point under the constraint (1.7) if and only if its extension (1.9) has a critical point under the constraint (1.10).

Now we are able to state the main result of this chapter. 
Theorem 2.2. Let $\Omega \subset \mathbb{R}^{n}$ be a bounded domain with Lipschitz boundary. There exists a sequence $\left(u_{k},-u_{k}\right)_{k \in \mathbb{N}}, u_{k} \in B V(\Omega)$, of pairs of critical points of (1.6) under the constraint (1.7) with $E\left(u_{k}\right)<\infty$ for all $k \in \mathbb{N}$. Moreover, for each critical point $u_{k} \in B V(\Omega)$ and any $1<q<\frac{n}{n-1}$ there exists a measurable selection $s_{k}(x) \in \operatorname{Sgn}\left(u_{k}(x)\right)$ for a.e. $x \in \Omega$ and a vector field $z_{k} \in L^{\infty}\left(\Omega, \mathbb{R}^{n}\right)$ with

$$
\begin{gathered}
\left\|z_{k}\right\|_{L^{\infty}}=1, \quad \operatorname{Div} z_{k} \in L^{q^{*}}(\Omega), \\
E\left(u_{k}\right)=-\int_{\Omega} u_{k} \operatorname{Div} z_{k} d x
\end{gathered}
$$

such that

$$
-\operatorname{Div} z_{k}=\lambda_{k} s_{k} \text { a.e. on } \Omega, \quad \lambda_{k}=E\left(u_{k}\right)
$$

We also have $\lambda_{k} \rightarrow \infty$ as $k \rightarrow \infty$.

Notice that critical points have to satisfy only a single Euler-Lagrange equation by this Theorem.

\subsection{Proof of Theorem 2.2}

In this section we carry out the proof of Theorem 2.2. After some preliminary considerations we apply Proposition 2.3 from below, which is due to Degiovanni \& Marzocchi [15], to our setting. We claim to verify the existence of a sequence of critical points of (1.9) under the constraint (1.10) which, by definition, are considered as critical points of (1.6) under the constraint (1.7). According to Lemma 2.1 it is sufficient to verify critical points of the functional $E+I_{K}$ on $L^{q}(\Omega)$. The functional $E+I_{K}$ is merely lower semicontinuous, since $E$ is lower semicontinuous, cf. Proposition 1.5, and $K$ is a closed set. Hence we have to determine critical points of the corresponding function $\mathscr{G}_{E+I_{K}}$ according to (2.5). More precisely, we show the existence of critical points of

$$
\mathscr{G}_{E+I_{K}}: \operatorname{epi}\left(E+I_{K}\right) \rightarrow \mathbb{R}, \quad \mathscr{G}_{E+I_{K}}(u, \xi)=\xi
$$

with

$$
\operatorname{epi}\left(E+I_{K}\right)=\left\{(u, \xi) \in L^{q}(\Omega) \times \mathbb{R} \mid u \in B V(\Omega) \cap K, E(u) \leq \xi\right\} \text {, }
$$


where epi $\left(E+I_{K}\right)$ is endowed with the metric according to (2.4) that is induced by the $L^{q}$-norm. In Lemma 2.5 below we see that (2.6) is satisfied in general and, thus, critical points of $\mathscr{G}_{E+I_{K}}$ are also critical points of $E+I_{K}$. But, before we formulate the details of the proof, let us introduce some notions.

Let $X$ be a metric space endowed with metric $m$ and let $A \subset X$ be a closed nonempty set. The category of $A$ in $X$, denoted by cat $(A, X)$, is defined as the least $k \in \mathbb{N}$ such that $A$ can be covered by $k$ open subsets of $X$, each of which is contractible in $X$. If no such $k$ exists, we set cat $(A, X)=\infty$. We also set $\operatorname{cat}(\emptyset, X)=0$.

A metric space $X$ is said to be weakly locally contractible if for every $u \in X$ there exists a neighborhood $U$ of $u$ which is contractible in $X$.

Let $X$ be a topological space and $A \subset X$. Then a continuous function $r: X \rightarrow A$ is a retraction if $r(a)=a$ for all $a \in A$. A subspace $A$ is called a retract of $X$ if such a retraction exists.

Let $F: X \rightarrow \mathbb{R} \cup\{\infty\}$ be a lower semicontinuous function defined on a metric space $X$ and let $c \in \mathbb{R}$. A sequence $\left(u_{k}\right)_{k \in \mathbb{N}}$ in $\mathcal{D}(F)$, cf. (2.3), is said to be a Palais-Smale sequence at level $c$ for $F$, if

$$
F\left(u_{k}\right) \rightarrow c \quad \text { and } \quad|d F|\left(u_{k}\right) \rightarrow 0
$$

We say that $F$ satisfies the Palais-Smale condition at level $c$, if every Palais-Smale sequence $\left(u_{k}\right)_{k \in \mathbb{N}}$ at level $c$ for $F$ has a convergent subsequence in $X$.

Let $A$ be a convex subset of a Banach space $X$ and $u \in A \cap K$ with $K$ as in (2.7). Then $A$ and $K$ are said to be transversal at $u$, if there exist $u_{-}, u_{+} \in A$ such that

$$
G^{0}\left(u, u_{-}-u\right)<0 \quad \text { and } \quad G^{0}\left(u, u-u_{+}\right)<0 .
$$

We claim to apply the following result, which can be found in Degiovanni \& Marzocchi [15, Theorem 3.10] and which is an adaptation of a classical result to a nonsmooth setting.

Proposition 2.3. Let $X$ be a weakly locally contractible complete metric space and let $F: X \rightarrow \mathbb{R}$ be a continuous function which is bounded from below and 
which satisfies the Palais-Smale condition at level $c$ for all $c \in \mathbb{R}$. Moreover, let

$$
\sup \{\operatorname{cat}(A, X) \mid A \subset X \text { compact }\}=\infty .
$$

Then $F$ has infinitely many critical points $\left(u_{k}\right)_{k \in \mathbb{N}}$ with critical values

$$
c_{k}=\inf _{A \in \mathcal{A}_{k}} \sup _{u \in A} F(u), \quad c_{k} \rightarrow \infty
$$

where

$$
\mathcal{A}_{k}=\{A \subset X \mid A \text { compact, } \operatorname{cat}(A, X) \geq k\},
$$

and $\sup _{X} F=\infty$.

In order to get a nontrivial setting for the application of Proposition 2.3 we have to reformulate our problem again. We transfer our considerations from the Banach space $Y$ to the projective space $\mathcal{P}_{Y}$ obtained from $Y$ by identifying every $u \in Y \backslash\{0\}$ with its antipodal point $-u$. The relation $u \sim v$, if $u=v$ or $u=-v$, is an equivalence relation and $\mathcal{P}_{Y}=(Y \backslash\{0\}) / \sim$ is the corresponding quotient space. We denote the elements of $\mathcal{P}_{Y}$ by $[u]$ and endow $\mathcal{P}_{Y}$ with the induced metric

$$
m_{\mathcal{P}_{Y}}([u],[v]):=\min \{m(\bar{u}, \bar{v}) \mid \bar{u} \in[u], \bar{v} \in[v]\}=\min \{m(u, v), m(u,-v)\} .
$$

We call a set $A \subset Y$ symmetric, if for each $u \in A$ also $-u \in A$. For a symmetric set $A \subset Y \backslash\{0\}$ we denote by $\mathcal{P}_{Y}(A)$ the corresponding set in the projective space $\mathcal{P}_{Y}$.

In our case $Y:=L^{q}(\Omega)$ and we denote the corresponding projective space by $\mathcal{P}$ for simplicity. Since $E$ and $G$ according to (1.9), (1.10) are even functionals, we can think of them as mappings from $\mathcal{P}$ to $\mathbb{R} \cup\{\infty\}$, i. e. without danger of confusion we set $E: \mathcal{P} \rightarrow \mathbb{R} \cup\{\infty\}$ and $G: \mathcal{P} \rightarrow \mathbb{R} \cup\{\infty\}$ as

$$
E([u]):=E(\bar{u}) \quad \text { and } \quad G([u]):=G(\bar{u})
$$

for any representative $\bar{u} \in[u]$. 
We intend to apply Proposition 2.3 to the map

$$
\mathscr{G}_{E+I_{K}}([u], \xi)=\xi
$$

defined on

$$
X:=\operatorname{epi}_{\mathcal{P}}\left(E+I_{K}\right)=\{([u], \xi) \in \mathcal{P}(B V(\Omega) \cap K) \times \mathbb{R} \mid E([u]) \leq \xi\}
$$

as a subset of the space $\mathcal{P} \times \mathbb{R}$ which is endowed with the metric

$$
m_{\mathcal{P}}(([u], \xi),([v], \mu))=\sqrt{m_{\mathcal{P}}([u],[v])^{2}+(\xi-\mu)^{2}}
$$

and epi $\operatorname{P}_{\mathcal{P}}\left(E+I_{K}\right)$ with the induced metric. Note that $0 \notin B V(\Omega) \cap K$ and $B V(\Omega) \cap K$ is a symmetric set. According to the following Lemma the critical points $([u], E([u]))$ of $\mathscr{G}_{E+I_{K}}$ on epi $\mathcal{P}_{\mathcal{P}}\left(E+I_{K}\right)$ correspond to pairs of critical points $((u, E(u)),(-u, E(-u)))$ of $\mathscr{G}_{E+I_{K}}$ on epi $\left(E+I_{K}\right)$.

Lemma 2.4. Let $Y$ be a Banach space with metric $m$, let $\mathcal{P}_{Y}$ be the corresponding projective space, and let $F: Y \rightarrow \mathbb{R} \cup\{\infty\}$ be a lower semicontinuous even functional. If $[u] \in \mathcal{P}_{Y}$ with $u \in \mathcal{D}(F)$ is a critical point of $F$ with respect to $\mathcal{P}_{Y}$, then $u$ and $-u$ are critical points of $F$ with respect to $Y$.

Proof. It is enough to show that $u$ is a critical point of $F$ for any fixed representative $\bar{u} \in[u]$, since $F$ is even. We assume first that $F$ is continuous on $Y$, which implies that $F$ is also continuous on $\mathcal{P}_{Y}$. Let us assume that

$$
|d F|^{\mathcal{P}_{Y}}([u])=0 \quad \text { and } \quad|d F|^{Y}(\bar{u})>0 \quad \text { for any } \bar{u} \in[u]
$$

Then, by the definition of the weak slope, there exist constants $\sigma>0, \delta>0$ and a continuous map $\mathcal{H}: B_{\delta}(\bar{u}) \times[0, \delta] \rightarrow Y$ such that for all $v \in B_{\delta}(\bar{u})$ and all $t \in[0, \delta]$

$$
m(\mathcal{H}(v, t), v) \leq t
$$

and

$$
F(\mathcal{H}(v, t)) \leq F(v)-\sigma t
$$

Since $\bar{u} \neq 0$, we achieve that $\operatorname{dist}\left(B_{\delta}(\bar{u}), B_{\delta}(-\bar{u})\right)>0$ and $\mathcal{H}(v, t) \neq 0$ for all 
$v \in B_{\delta}(\bar{u})$ and all $t \in[0, \delta]$ by choosing a smaller $\delta>0$ if necessary. We see that

$$
B_{\delta}([u]):=\mathcal{P}_{Y}\left(B_{\delta}(\bar{u}) \cup-B_{\delta}(\bar{u})\right)
$$

is a neighborhood of $[u]$ in $\mathcal{P}_{Y}$.

Now we define a deformation $\tilde{\mathcal{H}}: B_{\delta}([u]) \times[0, \delta] \rightarrow \mathcal{P}_{Y}$ by

$$
\tilde{\mathcal{H}}([v], t):=[\mathcal{H}(\bar{v}, t)]
$$

where the representative $\bar{v} \in[v]$ is chosen such that $\bar{v} \in B_{\delta}(\bar{u})$. We must show that $\tilde{\mathcal{H}}$ is continuous. Let $([v], t) \in B_{\delta}([u]) \times[0, \delta]$ and $\left(\left[v_{k}\right], t_{k}\right)_{k \in \mathbb{N}} \in B_{\delta}([u]) \times[0, \delta]$ with $m_{\mathcal{P}_{Y}}\left(([v], t),\left(\left[v_{k}\right], t_{k}\right)\right) \rightarrow 0$. Note that if we pick the representatives $\bar{v} \in[v]$, $\bar{v}_{k} \in\left[v_{k}\right]$ such that $\bar{v}, \bar{v}_{k} \in B_{\delta}(\bar{u})$, we have that $m\left((\bar{v}, t),\left(\bar{v}_{k}, t_{k}\right)\right) \rightarrow 0$, since $m\left(\bar{v},-\bar{v}_{k}\right) \geq \operatorname{dist}\left(B_{\delta}(\bar{u}), B_{\delta}(-\bar{u})\right)>0$. For $\bar{v}, \bar{v}_{k} \in B_{\delta}(\bar{u})$ and $t, t_{k} \in[0, \delta]$ we get

$$
\begin{aligned}
m_{\mathcal{P}_{Y}}\left(\tilde{\mathcal{H}}([v], t), \tilde{\mathcal{H}}\left(\left[v_{k}\right], t_{k}\right)\right)=m_{\mathcal{P}_{Y}}\left([\mathcal{H}(\bar{v}, t)],\left[\mathcal{H}\left(\bar{v}_{k}, t_{k}\right)\right]\right) \\
\quad=\min \left\{m\left(\mathcal{H}(\bar{v}, t), \mathcal{H}\left(\bar{v}_{k}, t_{k}\right)\right), m\left(\mathcal{H}(\bar{v}, t),-\mathcal{H}\left(\bar{v}_{k}, t_{k}\right)\right)\right\} \\
\leq m\left(\mathcal{H}(\bar{v}, t), \mathcal{H}\left(\bar{v}_{k}, t_{k}\right)\right) \rightarrow 0
\end{aligned}
$$

for $m_{\mathcal{P}_{Y}}\left(([v], t),\left(\left[v_{k}\right], t_{k}\right)\right) \rightarrow 0$, since $\mathcal{H}$ is continuous on $B_{\delta}(\bar{u}) \times[0, \delta]$.

Moreover,

$$
m_{\mathcal{P}_{Y}}(\tilde{\mathcal{H}}([v], t),[v])=m_{\mathcal{P}_{Y}}([\mathcal{H}(\bar{v}, t)],[v]) \leq m(\mathcal{H}(\bar{v}, t), \bar{v}) \leq t
$$

by $(2.11)$ and

$$
F(\tilde{\mathcal{H}}([v], t))=F([\mathcal{H}(\bar{v}, t)])=F(\mathcal{H}(\bar{v}, t)) \leq F(\bar{v})-\sigma t
$$

with a $\sigma>0$ by (2.12). This contradicts (2.10). Therefore $u$ and $-u$ are critical points of $F$ on $Y$.

Now let us assume, that $F: \mathcal{P}_{Y} \rightarrow \mathbb{R} \cup\{\infty\}$ is merely lower semicontinuous and has a critical point $[u] \in \mathcal{P}_{Y}$. Then, by definition, the function $\mathscr{G}_{F}: \operatorname{epi}_{\mathcal{P}_{Y}}(F) \rightarrow \mathbb{R}$ has a critical point $([u], F([u]))=([u], F(u))$. Since $\mathscr{G}_{F}$ is continuous, we can follow the previous argumentation step by step in the first 
component of the variable of $\mathscr{G}_{F}$. Thus, $\mathscr{G}_{F}$ must also have critical points $(u, F(u))$ and $(-u, F(u))$ on epi $(F)$. But then $F$ also has critical points $u$ and $-u$ on $Y$.

For the proof of Lemma 2.6 below we have to show transversality of the convex set $\mathcal{D}(E)=B V(\Omega)$ and $K$ in $L^{q}(\Omega)$ at each $u \in B V(\Omega) \cap K$.

Lemma 2.5. The sets $B V(\Omega)$ and $K$ are transversal in $L^{q}(\Omega)$ at each $u \in$ $B V(\Omega) \cap K$. Moreover, (2.6) is satisfied.

Proof. Let $u \in B V(\Omega) \cap K$. We define

$$
u_{-}:=0 \quad \text { and } \quad u_{+}:=2 u
$$

Then $u_{-}, u_{+} \in B V(\Omega)$ and, using Clarke [12, Proposition 2.1.2] and Proposition 1.6, we get

$$
\begin{aligned}
& G^{0}\left(u, u_{-}-u\right)=G^{0}(u, u\left.-u_{+}\right)=G^{0}(u,-u) \\
&=\max _{u_{G}^{*} \in \partial G(u)}-\left\langle u_{G}^{*}, u\right\rangle=-G(u)-1=-1,
\end{aligned}
$$

cf. [31, Proof of Theorem 4.6], i. e. transversality is satisfied.

By Degiovanni \& Schuricht [16, Theorem 3.4] it is enough to show transversality of the convex set $\mathcal{D}(E)=B V(\Omega)$ and $K$ in $L^{q}(\Omega)$ at each $u \in \mathcal{D}(E) \cap K$ for (2.6), as we just did.

Now we are able to prove the following result.

Lemma 2.6. The set epi $\mathcal{P}\left(E+I_{K}\right)$ is a complete weakly locally contractible metric space.

Proof. We show the assertion for the set epi $\left(E+I_{K}\right) \subset L^{q}(\Omega) \times \mathbb{R}$. Since $0 \notin \mathrm{epi}\left(E+I_{K}\right)$ and since with $(u, \xi) \in \mathrm{epi}\left(E+I_{K}\right)$ also $(-u, \xi) \in$ epi $\left(E+I_{K}\right)$, we can choose the neighborhoods of $(u, \xi)$ and $(-u, \xi)$ to be disjoint. Thus the result follows for epi $\mathcal{P}\left(E+I_{K}\right)$.

First we show that epi $\left(E+I_{K}\right) \subset L^{q}(\Omega) \times \mathbb{R}$ is a complete metric space. Let $\left(u_{j}, \xi_{j}\right)_{j \in \mathbb{N}} \in \operatorname{epi}\left(E+I_{K}\right)$ with $\left(u_{j}, \xi_{j}\right) \rightarrow(u, \xi)$ in $L^{q}(\Omega) \times \mathbb{R}$. By $(2.9)$ we have 
$u_{j} \in B V(\Omega) \cap K$ and $E\left(u_{j}\right) \leq \xi_{j}$ for all $j \in \mathbb{N}$. Since $B V(\Omega) \cap K$ is closed and $E$ is lower semicontinuous on $L^{q}(\Omega)$, cf. [31, Proposition 4.23], we get

$$
E(u) \leq \liminf _{j \rightarrow \infty} E\left(u_{j}\right) \leq \lim _{j \rightarrow \infty} \xi_{j}=\xi
$$

Then $(u, \xi) \in \operatorname{epi}\left(E+I_{K}\right)$ and therefore epi $\left(E+I_{K}\right)$ is complete.

We will show that epi $\left(E+I_{K}\right)$ is an ANR (absolute neighborhood retract), see Borsuk [6, p. 85] for definition. Moreover, we use [6, p. 99, Corollary 10.4], which states that for separable metric spaces the property of being an ANR is a local property. Since epi $\left(E+I_{K}\right)$ is separable, in our case it is enough to show, that every $(u, \xi) \in \operatorname{epi}\left(E+I_{K}\right)$ has a neighborhood in $L^{q}(\Omega) \times \mathbb{R}$, which is an ANR. This will be shown below. Then, by [6, p. 28; p. 87, Corollary 3.3], the space epi $\left(E+I_{K}\right)$ is weakly locally contractible.

(a) First, for each $u \in B V(\Omega) \cap K$, we construct a neighborhood of $u$ in $L^{q}(\Omega) \cap K$ which is a retract of a neighborhood of $u$ in $L^{q}(\Omega)$.

We use functions $u_{-}$and $u_{+}$as defined in the proof of Lemma 2.5. The directional derivative $G^{0}(u, z)$ is upper semicontinuous as a function of $(u, z)$, cf. Clarke [12, Proposition 2.1.1], therefore, using Lemma 2.5, we can find a $\delta>0$ such that for all $v, w \in B_{\delta}(u)$ (a neighborhood of $u$ in $L^{q}(\Omega)$ )

$$
G^{0}\left(w, u_{-}-v\right)<0 \quad \text { and } \quad G^{0}\left(w, v-u_{+}\right)<0 .
$$

By replacing $u_{-}$and $u_{+}$by $u+t_{0}\left(u_{-}-u\right)=\left(1-t_{0}\right) u$ and $u+t_{0}\left(u_{+}-u\right)=\left(1+t_{0}\right) u$ for a suitable $t_{0} \in(0,1]$ if necessary, we can assume that $u_{-}, u_{+} \in B_{\delta}(u)$ (we keep the notation for simplicity). Since $B_{\delta}(u)$ is convex, we get

$$
G^{0}\left(v+t\left(u_{-}-v\right), u_{-}-v\right)<0 \quad \text { and } \quad G^{0}\left(v+t\left(u_{+}-v\right), v-u_{+}\right)<0
$$

for all $v \in B_{\delta}(u)$ and all $t \in[0,1]$. By a property of the generalized directional derivative, cf. [12, Proposition 2.1.1], we have

$$
G^{0}\left(v+t\left(u_{+}-v\right), v-u_{+}\right)=(-G)^{0}\left(v+t\left(u_{+}-v\right), u_{+}-v\right),
$$


thus

$$
G^{0}\left(v+t\left(u_{-}-v\right), u_{-}-v\right)<0 \quad \text { and } \quad(-G)^{0}\left(v+t\left(u_{+}-v\right), u_{+}-v\right)<0 .
$$

Note that by [12, Proposition 2.1.2] we have

$$
G^{0}\left(v+t\left(u_{-}-v\right), u_{-}-v\right)=\max \left\{\left\langle\zeta^{*}, u_{-}-v\right\rangle \mid \zeta^{*} \in \partial G\left(v+t\left(u_{-}-v\right)\right)\right\}<0
$$

and

$$
(-G)^{0}\left(v+t\left(u_{+}-v\right), u_{+}-v\right)=\max \left\{\left\langle\zeta^{*}, u_{+}-v\right\rangle \mid \zeta^{*} \in \partial(-G)\left(v+t\left(u_{+}-v\right)\right)\right\}<0
$$

Now we apply [12, p.41, Lemma] to the Lipschitz continuous functions

$$
g_{-}(t):=G\left(v+t\left(u_{-}-v\right)\right) \quad \text { and } \quad-g_{+}(t):=-G\left(v+t\left(u_{+}-v\right)\right)
$$

and get that

$$
\partial g_{-}(t) \subset\left\{\left\langle\zeta^{*}, u_{-}-v\right\rangle \mid \zeta^{*} \in \partial G\left(v+t\left(u_{-}-v\right)\right)\right\}
$$

and

$$
\partial\left(-g_{+}\right)(t) \subset\left\{\left\langle\zeta^{*}, u_{+}-v\right\rangle \mid \zeta^{*} \in \partial(-G)\left(v+t\left(u_{+}-v\right)\right)\right\} .
$$

Thus $g_{-}$and $-g_{+}$are strictly decreasing on $[0,1]$. In particular

$$
g_{+}(t)=G\left(v+t\left(u_{+}-v\right)\right)
$$

is strictly increasing on $[0,1]$. Note that $g_{-}(1)=-t_{0}$ and $g_{+}(1)=t_{0}$. Thus, for all $v \in B_{\delta}(u)$ with $G(v) \geq 0$ there exists a unique $\tau_{-}(v) \in[0,1)$ such that

$$
g_{-}\left(\tau_{-}(v)\right)=G\left(v+\tau_{-}(v)\left(u_{-}-v\right)\right)=0
$$

and, analogously, for all $v \in B_{\delta}(u)$ with $G(v) \leq 0$ there exists a unique $\tau_{+}(v) \in$ $[0,1)$ such that

$$
g_{+}\left(\tau_{+}(v)\right)=G\left(v+\tau_{+}(v)\left(u_{+}-v\right)\right)=0 .
$$


The function

$$
\tau(v):= \begin{cases}\tau_{-}(v) & \text { for } G(v) \geq 0 \\ \tau_{+}(v) & \text { for } G(v) \leq 0\end{cases}
$$

is continuous on $B_{\delta}(u)$ and if $v \in B_{\delta}(u) \cap K$, then $\tau(v)=0$. We define a function $r: B_{\delta}(u) \rightarrow B_{\delta}(u) \cap K$ in the following way

$$
r(v):= \begin{cases}v+\tau(v)\left(u_{-}-v\right) & \text { for } G(v) \geq 0 \\ v+\tau(v)\left(u_{+}-v\right) & \text { for } G(v) \leq 0\end{cases}
$$

We observe that $r$ is continuous and if $v \in K$ then $r(v)=v$. Moreover, $r\left(B_{\delta}(u)\right)=$ $B_{\delta}(u) \cap K$ and $B_{\delta}(u) \cap K$ is a neighborhood of $u$ in $L^{q}(\Omega) \cap K$. Thus $r: B_{\delta}(u) \rightarrow$ $B_{\delta}(u) \cap K$ is a retraction. Note that if $v \in B V(\Omega)$, then also $r(v) \in B V(\Omega)$.

(b) Let now $(u, \xi) \in$ epi $\left(E+I_{K}\right)$, i. e. $u \in B V(\Omega) \cap K$ and $E(u) \leq \xi$. We construct a neighborhood of $(u, \xi)$ in epi $\left(E+I_{K}\right)$ which is a retract of a neighborhood of $(u, \xi)$ in epi $(E)$.

We consider the neighborhood $\left(B_{\delta}(u) \times \mathbb{R}\right) \cap$ epi $(E)$ of $(u, \xi)$ in epi $(E)$ and construct a retraction of it onto $\left(B_{\delta}(u) \times \mathbb{R}\right) \cap$ epi $\left(E+I_{K}\right)$. For all $(v, \mu) \in$ $\left(B_{\delta}(u) \times \mathbb{R}\right) \cap \operatorname{epi}(E)$ we define the continuous function

$$
\tilde{r}(v, \mu):=\left(r(v),(1-\tau(v)) \mu+\tau(v) E\left(u_{+}\right)\right) .
$$

If $v \in K$, then $\tau(v)=0$ and therefore

$$
\tilde{r}(v, \mu)=(v, \mu) \quad \text { on } \quad\left(B_{\delta}(u) \times \mathbb{R}\right) \cap \operatorname{epi}\left(E+I_{K}\right) .
$$

We show that $\tilde{r}(v, \mu) \in$ epi $\left(E+I_{K}\right)$. By definition of $r$ we have $r(v) \in B_{\delta}(u) \cap$ $B V(\Omega) \cap K$. Moreover, since $E$ is 1-homogeneous, we get

$$
E\left(u_{-}\right)=E\left(\left(1-t_{0}\right) u\right)=\left(1-t_{0}\right) E(u)<\left(1+t_{0}\right) E(u)=E\left(\left(1+t_{0}\right) u\right)=E\left(u_{+}\right) .
$$

If $G(v) \geq 0$ we deduce, by using convexity and 1-homogeneity of $E$,

$$
\begin{aligned}
E(r(v)) & =E\left(v+\tau(v)\left(u_{-}-v\right)\right) \\
& =E\left((1-\tau(v)) v+\tau(v) u_{-}\right)
\end{aligned}
$$




$$
\begin{aligned}
& \leq(1-\tau(v)) E(v)+\tau(v) E\left(u_{-}\right) \\
& \leq(1-\tau(v)) E(v)+\tau(v) E\left(u_{+}\right)
\end{aligned}
$$

and, analogously, for $G(v) \leq 0$ we get

$$
E(r(v)) \leq(1-\tau(v)) E(v)+\tau(v) E\left(u_{+}\right)
$$

Therefore

$$
E(r(v)) \leq(1-\tau(v)) \mu+\tau(v) E\left(u_{+}\right)
$$

i. e. $\tilde{r}(v, \mu) \in \operatorname{epi}\left(E+I_{K}\right)$ and

$$
\tilde{r}:\left(B_{\delta}(u) \times \mathbb{R}\right) \cap \operatorname{epi}(E) \rightarrow\left(B_{\delta}(u) \times \mathbb{R}\right) \cap \text { epi }\left(E+I_{K}\right)
$$

is a retraction. Thus, the neighborhood $\left(B_{\delta}(u) \times \mathbb{R}\right) \cap$ epi $\left(E+I_{K}\right)$ of $(u, \xi)$ in epi $\left(E+I_{K}\right)$ is a retract of the neighborhood $\left(B_{\delta}(u) \times \mathbb{R}\right) \cap$ epi $(E)$ of $(u, \xi)$ in epi $(E)$.

(c) Since $E$ is convex, epi $(E)$ is also convex. Then also $\left(B_{\delta}(u) \times \mathbb{R}\right) \cap$ epi $(E)$ is convex as intersection of convex sets and by Dugundji [21, Corollary 4.2] an ANR. Thus, by $(\mathrm{b})$, the neighborhood $\left(B_{\delta}(u) \times \mathbb{R}\right) \cap$ epi $\left(E+I_{K}\right)$ is a retract of an ANR and by [6, p. 87, Corollary 3.2] also an ANR.

Lemma 2.7. The functional $\mathscr{G}_{E+I_{K}}$ satisfies the Palais-Smale condition at level $c$ for any $c \in \mathbb{R}$ as function on epi $\mathcal{P}\left(E+I_{K}\right)$.

Proof. Let us first show that $E+I_{K}$ satisfies the Palais-Smale condition at level $c$ as a function on $L^{q}(\Omega)$. Let $\left(u_{j}\right)_{j \in \mathbb{N}} \subset B V(\Omega) \cap K$ be any Palais-Smale sequence at level $c$ for $E+I_{K}$, i. e.

$$
\left(E+I_{K}\right)\left(u_{j}\right) \rightarrow c \quad \text { and } \quad\left|d\left(E+I_{K}\right)\right|\left(u_{j}\right) \rightarrow 0
$$

Since $\left(u_{j}\right)_{j \in \mathbb{N}}$ is bounded in $B V(\Omega)$ by $\left(E+I_{K}\right)\left(u_{j}\right)<\infty,\left(u_{j}\right)_{j \in \mathbb{N}}$ is also bounded in $L^{q}(\Omega)$. Since $B V(\Omega)$ is compactly embedded in $L^{q}(\Omega)$, there exists a convergent subsequence of $\left(u_{j}\right)_{j \in \mathbb{N}}$ in $L^{q}(\Omega)$.

Now we show that $\mathscr{G}_{E+I_{K}}$ satisfies the Palais-Smale condition on epi $\left(E+I_{K}\right)$. 
Let $\left(u_{j}, \xi_{j}\right) \subset \operatorname{epi}\left(E+I_{K}\right)$ be a Palais-Smale sequence for $\mathscr{G}_{E+I_{K}}$ at level $c$, i. e.

$$
\xi_{j}=\mathscr{G}_{E+I_{K}}\left(u_{j}, \xi_{j}\right) \rightarrow c \text { and }\left|d \mathscr{G}_{E+I_{K}}\right|\left(u_{j}, \xi_{j}\right) \rightarrow 0 .
$$

Since (2.6) is satisfied, cf. Lemma 2.5, there exists a $j_{0} \in \mathbb{N}$ such that for all $j \geq j_{0}$ we have $\xi_{j}=\left(E+I_{K}\right)\left(u_{j}\right)$. Moreover, by the definition of the weak slope

$$
\left|d\left(E+I_{K}\right)\right|\left(u_{j}\right)=\frac{\left|d \mathscr{G}_{E+I_{K}}\right|\left(u_{j},\left(E+I_{K}\right)\left(u_{j}\right)\right)}{\sqrt{1-\left|d \mathscr{G}_{E+I_{K}}\right|\left(u_{j},\left(E+I_{K}\right)\left(u_{j}\right)\right)}} \rightarrow 0
$$

and therefore $\left(u_{j}\right)_{j \in \mathbb{N}}$ satisfies (2.13), i. e. $\left(u_{j}\right)_{j \in \mathbb{N}}$ is a Palais-Smale sequence for $E+I_{K}$ at level $c$. Since $E+I_{K}$ satisfies the Palais-Smale condition at level $c$, there exists a convergent subsequence of $\left(u_{j}\right)_{j \in \mathbb{N}}$ in $L^{q}(\Omega)$. Since $\left(\xi_{j}\right)_{j \in \mathbb{N}}$ is convergent by (2.14) in $\mathbb{R}$, we deduce that $\left(u_{j}, \xi_{j}\right)_{j \in \mathbb{N}}$ has a convergent subsequence in the complete metric space epi $\left(E+I_{K}\right)$.

Now we can easily transfer the result to $\mathscr{G}_{E+I_{K}}$ defined on epi $\mathcal{P}\left(E+I_{K}\right)$.

Lemma 2.8. We have

$$
\sup \left\{\operatorname{cat}\left(A, \operatorname{epi}_{\mathcal{P}}\left(E+I_{K}\right)\right) \mid A \subset \operatorname{epi}_{\mathcal{P}}\left(E+I_{K}\right) \text { compact }\right\}=\infty .
$$

Proof. The idea of the proof is to construct compact subsets of $\operatorname{epi}_{\mathcal{P}}\left(E+I_{K}\right)$ with arbitrarily large category.

In the proof of Proposition 2.6 we have shown that epi $\mathcal{P}_{\mathcal{P}}\left(E+I_{K}\right)$ is an ANR. In this case we can take closed covers instead of open covers in the definition of category (cf. Cornea et al. [13, Proposition 1.10]) and, in this proof, we use the definition of category by means of closed sets.

We fix any $k \in \mathbb{N}$ and linearly independent functions $v_{1}, \ldots, v_{k} \in B V(\Omega)$. We set

$$
V_{k}:=\operatorname{span}\left\{v_{i} \mid i=1, \ldots, k\right\} \cap K .
$$

By $S^{k-1} \subset \mathbb{R}^{k}$ we denote the $(k-1)$-dimensional unit sphere and $\mathbb{R}^{k}$ shall be endowed with the canonical basis $\left\{e_{i} \in \mathbb{R}^{k} \mid i=1, \ldots, k\right\}$. We construct a homeomorphism $\psi: S^{k-1} \rightarrow V_{k}$ by setting

$$
x=\sum_{i=1}^{k} x_{i} e_{i} \longmapsto \frac{\sum_{i=1}^{k} x_{i} v_{i}}{\left\|\sum_{i=1}^{k} x_{i} v_{i}\right\|_{L^{1}(\Omega)}} .
$$


We have

$$
\left\|\sum_{i=1}^{k} x_{i} v_{i}\right\|_{L^{1}(\Omega)} \geq C>0,
$$

since not all $x_{i}=0$, since the $v_{i}$ are linearly independent, and since

$$
x \mapsto\left\|\sum_{i=1}^{k} x_{i} v_{i}\right\|_{L^{1}(\Omega)}
$$

is a continuous function defined on the compact set $S^{k-1}$. We see that $\psi$ is a homeomorphism, since its inverse $\psi^{-1}: \psi\left(S^{k-1}\right) \rightarrow S^{k-1}$ is given by

$$
u=\sum_{i=1}^{k} \xi_{i} v_{i} \longmapsto \frac{\sum_{i=1}^{k} \xi_{i} e_{i}}{\sqrt{\sum_{i=1}^{k}\left|\xi_{i}\right|^{2}}}
$$

and $\psi^{-1}$ is continuous, since it is the inverse of a continuous function on a compact set. Clearly $\psi$ is odd, i. e. it satisfies $\psi(-x)=-\psi(x)$, and the set $\psi\left(S^{k-1}\right)$ is compact and symmetric.

For $u \in \psi\left(S^{k-1}\right)$ we obtain

$$
\begin{aligned}
E(u) & =E\left(\frac{\sum_{i=1}^{k} \xi_{i} v_{i}}{\left\|\sum_{i=1}^{k} \xi_{i} v_{i}\right\|_{L^{1}(\Omega)}}\right) \\
& =\frac{1}{\left\|\sum_{i=1}^{k} \xi_{i} v_{i}\right\|_{L^{1}(\Omega)}} E\left(\sum_{i=1}^{k} \xi_{i} v_{i}\right) \\
& =\frac{1}{\left\|\sum_{i=1}^{k} \xi_{i} v_{i}\right\|_{L^{1}(\Omega)}}\left(\int_{\Omega} d\left|D \sum_{i=1}^{k} \xi_{i} v_{i}\right|+\int_{\partial \Omega}\left|\sum_{i=1}^{k} \xi_{i} v_{i}\right| d \mathcal{H}^{n-1}\right) \\
& \leq \frac{1}{\left\|\sum_{i=1}^{k} \xi_{i} v_{i}\right\|_{L^{1}(\Omega)}} \sum_{i=1}^{k}\left|\xi_{i}\right|\left(\int_{\Omega} d\left|D v_{i}\right|+\int_{\partial \Omega}\left|v_{i}\right| d \mathcal{H}^{n-1}\right) \\
& (2.15){ }^{1} \sum_{i=1}^{k}\left|\xi_{i}\right| E\left(v_{i}\right) \\
& \leq \frac{k}{C} \max _{i=1, \ldots, k} E\left(v_{i}\right)=: C_{k} .
\end{aligned}
$$

Thus

$$
\sup _{u \in \psi\left(S^{k-1}\right)} E(u) \leq C_{k}
$$


for a constant $C_{k} \in \mathbb{R}$ depending only on $k$.

Let us now consider the real projective space $\mathcal{P}^{k-1}$ which is defined as the projective space according to $\mathbb{R}^{k} \backslash\{0\}$. By Zeidler [41, p. 347] we know that

$$
k=\operatorname{cat}\left(\mathcal{P}\left(S^{k-1}\right), \mathcal{P}\left(S^{k-1}\right)\right) .
$$

By a straightforward argument following from the definition of category one readily obtains that

$$
\text { cat }\left(\mathcal{P}\left(S^{k-1}\right), \mathcal{P}\left(S^{k-1}\right)\right)=\operatorname{cat}\left(\mathcal{P}^{k-1}, \mathcal{P}^{k-1}\right) \text {. }
$$

Now we use Fadell [23, Proposition 2.10] with $S^{k-1}$ and $\psi\left(S^{k-1}\right)$ as subsets of $\mathbb{R}^{k} \backslash\{0\}$ and $L^{q}(\Omega) \backslash\{0\}$, respectively, and get

$$
\text { cat }\left(\mathcal{P}^{k-1}, \mathcal{P}^{k-1}\right)=\operatorname{cat}\left(\mathcal{P}\left(\psi\left(S^{k-1}\right)\right), \mathcal{P}\right) \text {. }
$$

Note that we get equality, since $\psi$ is a homeomorphism.

Moreover, by the definition of category, we get

$$
\text { cat }\left(\mathcal{P}\left(\psi\left(S^{k-1}\right)\right), \mathcal{P}\right) \leq \operatorname{cat}\left(\mathcal{P}\left(\psi\left(S^{k-1}\right)\right), \mathcal{P}(B V(\Omega) \cap K)\right)
$$

since each covering of $\mathcal{P}\left(\psi\left(S^{k-1}\right)\right)$ by closed sets in $\mathcal{P}(B V(\Omega) \cap K)$ is always also a covering of $\mathcal{P}\left(\psi\left(S^{k-1}\right)\right)$ by closed sets in $\mathcal{P}$.

With the constant $C_{k}$ as defined in (2.16) we now assume that

$$
\text { cat }\left(\mathcal{P}\left(\psi\left(S^{k-1}\right)\right) \times\left\{C_{k}\right\}, \operatorname{epi}_{\mathcal{P}}\left(E+I_{K}\right)\right)=m
$$

for some $m \in \mathbb{N}$, i.e. there exists a covering of the form

$$
\mathcal{P}\left(\psi\left(S^{k-1}\right)\right) \times\left\{C_{k}\right\} \subset \bigcup_{i=1}^{m} \mathcal{P}\left(B_{k, i}\right) \times\left\{C_{k}\right\}
$$

with closed, symmetric, and contractible sets $B_{k, i} \subset B V(\Omega) \cap K$ such that

$$
\mathcal{P}\left(B_{k, i}\right) \times\left\{C_{k}\right\} \subset \operatorname{epi}_{\mathcal{P}}\left(E+I_{K}\right) .
$$


Note that $\left\{C_{k}\right\}$ is a covering of itself and contractible in itself. But then we also have

$$
\mathcal{P}\left(\psi\left(S^{k-1}\right)\right) \subset \bigcup_{i=1}^{m} \mathcal{P}\left(B_{k, i}\right)
$$

and therefore

$$
\begin{aligned}
\operatorname{cat}\left(\mathcal{P}\left(\psi\left(S^{k-1}\right)\right)\right. & , \mathcal{P}(B V(\Omega) \cap K)) \\
\leq & \operatorname{cat}\left(\mathcal{P}\left(\psi\left(S^{k-1}\right)\right) \times\left\{C_{k}\right\}, \operatorname{epi}_{\mathcal{P}}\left(E+I_{K}\right)\right)
\end{aligned}
$$

By analogous arguments we even get equality.

Combining equations (2.17), (2.18), (2.19), (2.20), and (2.21), we get

$$
\begin{aligned}
k & =\operatorname{cat}\left(\mathcal{P}\left(S^{k-1}\right), \mathcal{P}\left(S^{k-1}\right)\right) \\
& =\operatorname{cat}\left(\mathcal{P}^{k-1}, \mathcal{P}^{k-1}\right) \\
& =\operatorname{cat}\left(\mathcal{P}\left(\psi\left(S^{k-1}\right)\right), \mathcal{P}\right) \\
& \leq \operatorname{cat}\left(\mathcal{P}\left(\psi\left(S^{k-1}\right)\right), \mathcal{P}(B V(\Omega) \cap K)\right) \\
& =\operatorname{cat}\left(\mathcal{P}\left(\psi\left(S^{k-1}\right)\right) \times\left\{C_{k}\right\}, \operatorname{epi} \mathcal{P}\left(E+I_{K}\right)\right) .
\end{aligned}
$$

Since $k \in \mathbb{N}$ can be chosen arbitrarily large and since $\mathcal{P}\left(\psi\left(S^{k-1}\right)\right) \times\left\{C_{k}\right\} \subset$ epi $\left(E+I_{K}\right)$ by $(2.16)$, we have thus shown that epi $\mathcal{P}\left(E+I_{K}\right)$ contains compact sets of arbitrarily large category.

For the derivation of equation (2.8) in Theorem 2.2 we use a suitable Lagrange multiplier rule derived in Degiovanni \& Schuricht [16, Corollary 3.6] and the characterization of the subdifferentials of $E$ and $G$ as given in Propositions 1.5 and 1.6.

Proposition 2.9. Let $E: X \rightarrow \mathbb{R}$ be a lower semicontinuous convex function and let $G: X \rightarrow \mathbb{R}$ be locally Lipschitz continuous. Let $u \in \mathcal{D}(E) \cap K$ be a critical point of $E$ under the constraint $K$. If $\mathcal{D}(E)$ and $K$ are transversal at $u$ in $L^{q}(\Omega)$, then $\partial E(u) \neq \emptyset$ and there exist

$$
u_{E}^{*} \in \partial E(u), \quad u_{G}^{*} \in \partial G(u), \quad \lambda \in \mathbb{R}
$$


such that

$$
u_{E}^{*}+\lambda u_{G}^{*}=0 .
$$

Now we combine the provided tools in the final proof.

Proof of Theorem 2.2. We use Lemma 2.6, 2.7 and 2.8 and Proposition 2.3 in order to get the existence of infinitely many critical points $\left([u]_{k}, \xi_{k}\right)_{k \in \mathbb{N}}$ of $\mathscr{G}_{E+I_{K}}$ on the space epi $\mathcal{P}_{\mathcal{P}}\left(E+I_{K}\right)$. Note that $\mathscr{G}_{E+I_{K}}$ is bounded from below by $E(u) \geq 0$.

By going back to epi $\left(E+I_{K}\right)$ we thus have the existence of infinitely many pairs $\left(\left(u_{k}, \xi_{k}\right),\left(-u_{k}, \xi_{k}\right)\right)_{k \in \mathbb{N}}$ of critical points for $\mathscr{G}_{E+I_{K}}$ on epi $\left(E+I_{K}\right)$ by Lemma 2.4 . By Lemma 2.5 we know that $\mathscr{G}_{E+I_{K}}$ satisfies (2.6) and, therefore, the critical points of $\mathscr{G}_{E+I_{K}}$ are in fact $\left(\left(u_{k}, E\left(u_{k}\right)\right),\left(-u_{k}, E\left(u_{k}\right)\right)\right)_{k \in \mathbb{N}}$ and thus $\left(u_{k},-u_{k}\right)_{k \in \mathbb{N}}$ are critical points of $E+I_{K}$. Since $u_{k} \in B V(\Omega) \cap K$ for each $k \in \mathbb{N}$, we also get $E\left(u_{k}\right)<\infty$.

Now, since $B V(\Omega)$ and $K$ are transversal by Lemma 2.5, we may apply Proposition 2.9 by using Propositions 1.5 and 1.6. Then, for each critical point $u_{k} \in$ $B V(\Omega)$ there exists a measurable selection $s_{k}(x) \in \operatorname{Sgn}\left(u_{k}(x)\right)$ for a.e. $x \in \Omega$ and a vector field $z_{k} \in L^{\infty}\left(\Omega, \mathbb{R}^{n}\right)$ with

$$
\begin{gathered}
\left\|z_{k}\right\|_{L^{\infty}}=1, \quad \operatorname{Div} z_{k} \in L^{q^{*}}(\Omega), \\
E\left(u_{k}\right)=-\int_{\Omega} u_{k} \operatorname{Div} z_{k} d x
\end{gathered}
$$

such that

$$
-\operatorname{Div} z_{k}=\lambda_{k} s_{k} \text { a. e. on } \Omega \text {. }
$$

Moreover, by multiplying this equation with $u_{k}$ and integrating over $\Omega$ we get

$$
E\left(u_{k}\right)=-\int_{\Omega} u_{k} \operatorname{Div} z_{k} d x=\lambda_{k} \int_{\Omega} u_{k} s_{k} d x=\lambda_{k} .
$$

Going back to Proposition 2.3 and using that $\lambda_{k}=E\left(u_{k}\right)=\mathscr{G}_{E+I_{K}}\left(u_{k}, E\left(u_{k}\right)\right)$ we deduce that $\lambda_{k} \rightarrow \infty$ as $k \rightarrow \infty$. 


\section{Chapter 3}

\section{Necessary Condition for First Eigensolution by Inner Variations}

The purpose of the present chapter is the derivation of a necessary condition for the first eigensolution of the 1-Laplace operator, which is substantially different from the Euler-Lagrange equation as stated in Theorem 1.7. In the classical calculus of variations such a condition can be obtained from the variation of the domain $\Omega$. It turns out to be useful, if the solution is nonsmooth.

\subsection{Motivation and Formulation of the Neces- sary Condition}

In the classical calculus of variations we consider a variational integral

$$
\int_{\Omega} F(x, u(x), D u(x)) d x,
$$

where $F: \Omega \times \mathbb{R} \times \mathbb{R}^{n} \rightarrow \mathbb{R},(x, u, p) \mapsto F(x, u, p)$ is of class $C^{1}$. For every minimizer $u$ of the integral with respect to fixed boundary conditions, the variation at $u$ in the direction of any $\xi \in C_{0}^{\infty}(\Omega)$ has to vanish, i. e. for

$$
\varphi(t):=\int_{\Omega} F(x, u(x)+t \xi(x), D u(x)+t D \xi(x)) d x
$$


we have

$$
\varphi^{\prime}(0)=0
$$

This way we obtain the Euler-Lagrange equation for $u$ (in the weak form) given by

$$
\int_{\Omega} \xi \frac{\partial}{\partial u} F-\left\langle\frac{\partial}{\partial p} F, D \xi\right\rangle d x=0
$$

where the argument of $F$ is $(x, u(x), D u(x))$.

A similar relation can be obtained from variations of the variable $x$. In this case we define

$$
\Phi(t):=\int_{\Omega} F(y(x, t), u(y(x, t)), D u(y(x, t))) d x,
$$

with $y(x, t)=x+t \xi(x)$. Here $\xi \in C_{0}^{\infty}\left(\Omega, \mathbb{R}^{n}\right)$, and the interval $\left(-t_{0}, t_{0}\right) \subset \mathbb{R}$ of definition of $t$ is chosen such that $y: \Omega \times\left(-t_{0}, t_{0}\right) \rightarrow \Omega$ and $y(\cdot, t)$ is a $C^{\infty}$ diffeomorphism for any fixed $t \in\left(-t_{0}, t_{0}\right)$. Again, the variation of the integral has to vanish at a minimizer $u$ in direction of any $\xi \in C_{0}^{\infty}\left(\Omega, \mathbb{R}^{n}\right)$, i. e.

$$
\Phi^{\prime}(0)=0
$$

cf. Giaquinta \& Hildebrandt [27, p. 147], which is given by

$$
\int_{\Omega}\left\langle\frac{\partial}{\partial p} F, D \xi D u\right\rangle-\left\langle\frac{\partial}{\partial x} F, \xi\right\rangle-F \operatorname{div} \xi d x=0
$$

where the argument of $F$ is $(x, u(x), D u(x))$. Thus any minimizer $u \in C^{1}(\Omega)$ satisfies both the Euler-Lagrange equation and the necessary condition (3.1) obtained by inner variations. If $u \in C^{2}(\Omega)$ satisfies the Euler-Lagrange equation, then (3.1) is not helpful, since in this case it is also satisfied. However, if $u$ is less regular and satisfies the Euler-Lagrange equation, it must not satisfy (3.1). In this case we possibly get an additional necessary condition for the minimizer of the variational integral.

Let us consider the minimization problem belonging to the $p$-Laplace operator for $p \in(1, \infty)$ as defined in $(1.1),(1.2)$. Recall that both $E_{p}$ and $G_{p}$ are smooth functionals and the minimizer $u$ of $(1.1),(1.2)$ is in $C_{\mathrm{loc}}^{1, \alpha}(\Omega)$, cf. Theorem 1.3. 
Therefore the minimizer has to satisfy

$$
\int_{\Omega}\left\langle|D u|^{p-2} D u, D \xi D u\right\rangle-|D u|^{p} \operatorname{div} \xi d x=-\lambda \int_{\Omega}|u|^{p} \operatorname{div} \xi d x
$$

for all $\xi \in C_{0}^{\infty}\left(\Omega, \mathbb{R}^{n}\right)$ as we obtain by a straightforward calculation of (3.1) combined with the classical Lagrange multiplier rule. Note that since $u \notin C^{2}(\Omega)$ in general, equation (3.2) must not be equivalent to the Euler-Lagrange equation.

The formal limit of (3.2) for $p \rightarrow 1$ is

$$
\int_{\Omega}\left(\left\langle\frac{D u}{|D u|}, D \xi \frac{D u}{|D u|}\right\rangle-\operatorname{div} \xi\right)|D u| d x=-\lambda \int_{\Omega}|u| \operatorname{div} \xi d x,
$$

which is not well defined if we keep in mind that first eigenfunctions of the 1-Laplace operator might be piecewise constant, cf. Theorem 1.16. The derivation of a suitable substitute of equation (3.3) confronts us with the same difficulties as the derivation of the Euler-Lagrange equation in the case $p=1$. Since $E$ and $G$ as defined in (1.6), (1.7) are not smooth, we cannot use the classical Lagrange multiplier rule for the derivation of a necessary condition. Thus we must first provide a suitable version, which is applicable to our setting.

Let us now state the main result of this chapter, which gives us a suitable substitute for equation (3.3). For $u \in B V(\Omega)$ let $z \in L^{1}\left(\Omega, \mathbb{R}^{n}\right)$ denote the vector field satisfying

$$
|z(x)|=1 \quad \text { and } \quad D u=z|D u| \quad|D u| \text {-a.e. on } \Omega \text {, }
$$

according to the polar decomposition of the measure $(D u)$, cf. Ambrosio et al. [2, Corollary 1.29]. Note that by $D \xi z$ we mean the multiplication $D \xi(x) z(x)$ of the matrix $D \xi(x) \in \mathbb{R}^{n \times n}$ and the vector $z(x) \in \mathbb{R}^{n}$.

Theorem 3.1. Let $\Omega \subset \mathbb{R}^{n}$ be a bounded domain with Lipschitz boundary and $u \in B V(\Omega)$ a minimizer of (1.6) under the constraint (1.7). Then for each $\xi \in C_{0}^{\infty}\left(\Omega, \mathbb{R}^{n}\right)$ we have

$$
\int_{\Omega}\langle z, D \xi z\rangle-\operatorname{div} \xi d|D u|=-\lambda \int_{\Omega}|u| \operatorname{div} \xi d x
$$


with

$$
\lambda=E(u)=h(\Omega) .
$$

Thus, similar to Theorem 1.7 the vector field $z$ is a substitute for the formal expression $D u /|D u|$ in (3.3). The main difference of this $z$ to the one involved in the Euler-Lagrange equation is, that it is specified merely $|D u|$-a. e. on $\Omega$. Note that by Theorem 1.15 this $\lambda$ coincides with the first eigenvalue of the 1-Laplace operator.

We demonstrate how the new necessary condition can be exploited on a model example. Recall that we have constructed a continuum of solutions for the single Euler-Lagrange equation in Section 2.1. Hypothetically these functions could be minimizers of (1.6), (1.7), since minimizers satisfy the single Euler-Lagrange equation in particular. Now we apply our new necessary condition obtained by inner variations. More precisely, let us consider $B V(\Omega)$-functions

$$
v(x):=\frac{1}{|B|} \chi_{B}(x)
$$

for a ball $B=B_{r}\left(x_{0}\right) \in \mathbb{R}^{n}$ with $\bar{B} \subset \Omega$, cf. Figure 2.1. By (3.5) $v$ must satisfy

$$
\int_{\Omega}\langle z, D \xi z\rangle-\operatorname{div} \xi d|D v|=-\lambda \int_{\Omega}|v| \operatorname{div} \xi d x
$$

for any $\xi \in C_{0}^{\infty}\left(\Omega, \mathbb{R}^{n}\right)$ and $\lambda=E(v)=n / r$, cf. (1.18). We pick a $\xi \in C_{0}^{\infty}\left(\Omega, \mathbb{R}^{n}\right)$ such that $\xi(x)=x$ on a set which contains $\bar{B}$ but does not touch $\partial \Omega$. Then we have

$$
D \xi(x)=\mathrm{id} \text { and } \operatorname{div} \xi(x)=n \text { on } \bar{B} \text {. }
$$

Moreover, we know that $|D v|=\frac{1}{|B|} \mathcal{H}^{n-1}\lfloor\partial B$, cf. Evans \& Gariepy [22, p. 169]. Thus we get for the left hand side

$$
\begin{aligned}
\int_{\Omega}\langle z, D \xi z\rangle- & \operatorname{div} \xi d|D v|=\frac{1}{|B|} \int_{\partial B}\langle z, z\rangle-n d \mathcal{H}^{n-1} \\
= & \frac{1}{|B|} \int_{\partial B}(1-n) d \mathcal{H}^{n-1}=\frac{|\partial B|}{|B|}(1-n)=\frac{n}{r}(1-n)
\end{aligned}
$$


and for the right hand side

$$
-\lambda \int_{\Omega}|v| \operatorname{div} \xi d x=-\frac{n}{r} \frac{1}{|B|} \int_{B} n d x=-\frac{n^{2}}{r},
$$

which implies the contradiction

$$
n-1=n .
$$

Of course we are aware of the fact that such functions $v$ cannot be minimizers of (1.6), (1.7), since a disc $B$ which does not touch $\partial \Omega$ cannot minimize the Cheeger quotient, cf. Section 1.2. But these functions may be critical points of $E$ under the constraint $G(v)=0$. Since we expect that Theorem 3.1 remains true also for critical points of (1.6), (1.7) (if we take $\lambda=E(u)$ and disregard $\lambda=h(\Omega)$ ), we could rule out such functions.

\subsection{Proof of the Necessary Condition}

First of all, let us state a Lagrange multiplier rule which is applicable to our setting.

Proposition 3.2. Let $X$ be a linear space, $D \subset X$ with $0 \in D$ and $\mathcal{E}, \mathcal{G}: D \rightarrow \mathbb{R}$. Assume that

(i) $\mathcal{E}(0) \leq \mathcal{E}(\eta)$ for every $\eta \in D$ with $\mathcal{G}(\eta)=0$,

(ii) for every linear subspace $Y \subset X$ with $\operatorname{dim} Y \leq 2$, we have $0 \in \operatorname{int}_{Y}(D \cap Y)$,

(iii) directional derivatives $\delta \mathcal{E}(\cdot, \xi), \delta \mathcal{G}(\cdot, \xi): \operatorname{int}_{Y}(D \cap Y) \rightarrow \mathbb{R}$ exist and are continuous on $\operatorname{int}_{Y}(D \cap Y)$ for all $\xi \in X$ and $Y$ as in (ii),

(iv) there exists a $\xi_{0} \in X$ such that $\delta \mathcal{G}\left(0, \xi_{0}\right) \neq 0$.

Then there exists $a \lambda \in \mathbb{R}$ such that

$$
\delta \mathcal{E}(0, \xi)=\lambda \delta \mathcal{G}(0, \xi)
$$

for any $\xi \in X$. 
Proof. If $\operatorname{dim} X \leq 2$, the assertion is the classical Lagrange multiplier rule. Otherwise, let $\xi \in X$ and let $Y$ be the linear subspace spanned by $\xi$ and $\xi_{0}$. By the usual Lagrange multiplier rule, we find a $\lambda_{Y} \in \mathbb{R}$ such that

$$
\delta \mathcal{E}(0, \zeta)=\lambda_{Y} \delta \mathcal{G}(0, \zeta)
$$

for every $\zeta \in Y$. If we choose $\zeta=\xi_{0}$, we get

$$
\lambda_{Y}=\frac{\delta \mathcal{E}\left(0, \xi_{0}\right)}{\delta \mathcal{G}\left(0, \xi_{0}\right)},
$$

and therefore

$$
\delta \mathcal{E}(0, \zeta)=\frac{\delta \mathcal{E}\left(0, \xi_{0}\right)}{\delta \mathcal{G}\left(0, \xi_{0}\right)} \delta \mathcal{G}(0, \zeta)
$$

for each $\zeta \in Y$. Now we choose $\zeta=\xi$, and since $\xi \in X$ has been chosen arbitrarily, we conclude that

$$
\delta \mathcal{E}(0, \xi)=\frac{\delta \mathcal{E}\left(0, \xi_{0}\right)}{\delta \mathcal{G}\left(0, \xi_{0}\right)} \delta \mathcal{G}(0, \xi)
$$

for all $\xi \in X$. The assertion follows with $\lambda=\delta \mathcal{E}\left(0, \xi_{0}\right) / \delta \mathcal{G}\left(0, \xi_{0}\right)$.

We define the linear space $X:=C_{0}^{\infty}\left(\Omega, \mathbb{R}^{n}\right)$ endowed with the $C^{1}$-norm and

$$
D:=\{\eta \in X \mid(\mathrm{id}+\eta): \Omega \rightarrow \Omega \text { is a diffeomorphism }\}
$$

Then $0 \in D$, since id $: \Omega \rightarrow \Omega$ is a diffeomorphism. We define further

$$
\begin{gathered}
w(x):=u(x+\eta(x)) \quad \text { for any } \eta \in D, \\
\mathcal{E}: D \rightarrow \mathbb{R}, \quad \mathcal{E}(\eta):=\int_{\Omega} d|D w|
\end{gathered}
$$

and

$$
\mathcal{G}: D \rightarrow \mathbb{R}, \quad \mathcal{G}(\eta):=\int_{\Omega}|w| d x-1
$$

We will prove below, that $w \in B V(\Omega)$, since otherwise (3.8) is not well defined. Let $Y \subset X$ be a linear subspace with $\operatorname{dim} Y \leq 2$, i. e. there exist fixed $\eta_{1}, \eta_{2} \in X$ such that

$$
Y=\left\{\eta \in X \mid \eta=\alpha \eta_{1}+\beta \eta_{2}, \alpha, \beta \in \mathbb{R}\right\}
$$


In order to show (iii) of Proposition 3.2 we must calculate directional derivatives $\delta \mathcal{E}(\eta, \xi), \delta \mathcal{G}(\eta, \xi)$ for $\eta \in D \cap Y$ and $\xi \in X$. Thus we consider deformations

$$
\tilde{y}: \Omega \times \mathbb{R}^{3} \rightarrow \mathbb{R}^{n}, \quad \tilde{y}(x, \alpha, \beta, t):=x+\alpha \eta_{1}(x)+\beta \eta_{2}(x)+t \xi(x)
$$

with fixed $\eta_{1}, \eta_{2}, \xi \in X$ and functions

$$
v(x)=u(\tilde{y}(x, \alpha, \beta, t))
$$

and calculate

$$
\left.\frac{d}{d t} \mathcal{E}(\eta+t \xi)\right|_{t=0}=\left.\frac{d}{d t} \int_{\Omega} d|D v|\right|_{t=0} \quad \text { and }\left.\quad \frac{d}{d t} \mathcal{G}(\eta+t \xi)\right|_{t=0}=\left.\frac{d}{d t} \int_{\Omega}|v| d x\right|_{t=0} .
$$

Note that $\tilde{y} \in C^{\infty}\left(\Omega \times \mathbb{R}^{3}, \mathbb{R}^{n}\right)$. We use the notation

$$
D \tilde{y}(x, \alpha, \beta, t):=\frac{\partial}{\partial x} \tilde{y}(x, \alpha, \beta, t)
$$

for the partial derivative of $\tilde{y}$ with respect to $x$. We must show that $\alpha \eta_{1}+\beta \eta_{2}+t \xi \in$ $D$ for sufficiently small $\alpha, \beta, t$.

Lemma 3.3. There exist $\alpha_{0}, \beta_{0}, t_{0} \in \mathbb{R}^{+}$such that

$$
\tilde{y}: \Omega \times\left(-\alpha_{0}, \alpha_{0}\right) \times\left(-\beta_{0}, \beta_{0}\right) \times\left(-t_{0}, t_{0}\right) \rightarrow \Omega
$$

and $\tilde{y}(\cdot, \alpha, \beta, t)$ is a $C^{\infty}$-diffeomorphism for any fixed $\alpha \in\left(-\alpha_{0}, \alpha_{0}\right), \beta \in\left(-\beta_{0}, \beta_{0}\right)$, $t \in\left(-t_{0}, t_{0}\right)$. The partial derivative $D \tilde{y}(x, \alpha, \beta, t)$ is regular and there exists a constant $c \in \mathbb{R}^{+}$with

$$
\operatorname{det} D \tilde{y}(x, \alpha, \beta, t) \geq c>0
$$

for any fixed $(x, \alpha, \beta, t) \in \Omega \times\left(-\alpha_{0}, \alpha_{0}\right) \times\left(-\beta_{0}, \beta_{0}\right) \times\left(-t_{0}, t_{0}\right)$.

Proof. We denote the compact supports of $\eta_{1}, \eta_{2}, \xi$ by $K_{\eta_{1}}, K_{\eta_{2}}, K_{\xi}$ and set

$$
K:=K_{\eta_{1}} \cup K_{\eta_{2}} \cup K_{\xi}
$$

We extend $\tilde{y}$ on $\mathbb{R}^{n} \times \mathbb{R}^{3}$ by setting

$$
\tilde{y}(x, \alpha, \beta, t)=x
$$


for all $x \in \mathbb{R}^{n} \backslash \Omega$, i. e. we extend $\eta_{1}, \eta_{2}, \xi$ by 0 on $\mathbb{R}^{n}$. Since we have $\tilde{y}(x, \alpha, \beta, t)=$ $x$ on $\Omega \backslash K$ anyway, we get $\tilde{y} \in C^{\infty}\left(\mathbb{R}^{n} \times \mathbb{R}^{3}, \mathbb{R}^{n}\right)$.

Further we define a $C^{\infty}$-function $F: \mathbb{R}^{n} \times \mathbb{R}^{3} \times \mathbb{R}^{n} \rightarrow \mathbb{R}^{n}$ by

$$
F(y, \alpha, \beta, t, x):=\tilde{y}(x, \alpha, \beta, t)-y .
$$

We have $F\left(y_{1}, 0,0,0, y_{1}\right)=0$ for any fixed $y_{1} \in \mathbb{R}^{n}$ and we look for a function $\tilde{x}$ with $F(y, \alpha, \beta, t, \tilde{x}(y, \alpha, \beta, t))=0$ on a neighborhood of $\left(y_{1}, 0,0,0, y_{1}\right)$. The partial derivative $\frac{\partial}{\partial x} F$ exists everywhere on $\mathbb{R}^{n} \times \mathbb{R}^{3} \times \mathbb{R}^{n}$ and

$$
\frac{\partial}{\partial x} F\left(y_{1}, 0,0,0, y_{1}\right)=\mathrm{id}
$$

is bijective for any $y_{1} \in \mathbb{R}$. Then, by the implicit function theorem, cf. [40, Theorem 4.B], there exists a unique function $\tilde{x}$ defined on a neighborhood of $\left(y_{1}, 0,0,0\right)$ with

$$
F(y, \alpha, \beta, t, \tilde{x}(y, \alpha, \beta, t))=0 .
$$

Without loss of generality we can assume, that the neighborhood of $\left(y_{1}, 0,0,0\right)$ has the structure

$$
U_{1}:=Y_{1} \times\left(-\alpha_{1}\left(y_{1}\right), \alpha_{1}\left(y_{1}\right)\right) \times\left(-\beta_{1}\left(y_{1}\right), \beta_{1}\left(y_{1}\right)\right) \times\left(-t_{1}\left(y_{1}\right), t_{1}\left(y_{1}\right)\right) .
$$

Thus $\tilde{x}(\cdot, \alpha, \beta, t)$ is the inverse of $\tilde{y}(\cdot, \alpha, \beta, t)$ on $Y_{1}$ for a fixed $y_{1} \in \mathbb{R}^{n}$ and $\tilde{x} \in C^{\infty}\left(U_{1}, \mathbb{R}^{n}\right)$. Note that for all $y_{1} \in \mathbb{R}^{n} \backslash K$ we can set

$$
\alpha_{1}\left(y_{1}\right)=\beta_{1}\left(y_{1}\right)=t_{1}\left(y_{1}\right)=\infty,
$$

i. e. for such $y_{1}$ we have

$$
U_{1}=Y_{1} \times \mathbb{R} \times \mathbb{R} \times \mathbb{R} .
$$

To establish the global existence of the inverse $\tilde{x}(\cdot, \alpha, \beta, t)$ we use the global inverse mapping theorem (see Zeidler [40, Theorem 4.G]). We have shown that $\tilde{y}(\cdot, \alpha, \beta, t)$ is a $C^{\infty}$-diffeomorphism on $\mathbb{R}^{n}$ locally. In this case it is also a $C^{\infty}$ diffeomorphism globally if it is proper and in our case it is proper, if it is weakly coercive, cf. [40, Example 4.42]. For each $y_{1} \in K$ the function $\tilde{x}$ exists on a 
neighborhood $U_{1}$ of $\left(y_{1}, 0,0,0\right)$ as defined in (3.12). Since $K$ is a compact set and

$$
K \subset \bigcup_{y_{1} \in K} Y_{1}
$$

we can pick

$$
\begin{aligned}
\alpha_{0} & :=\min _{y_{1} \in \mathbb{R}^{n}} \alpha_{1}\left(y_{1}\right)=\min _{y_{1} \in K} \alpha_{1}\left(y_{1}\right), \\
\beta_{0} & :=\min _{y_{1} \in \mathbb{R}^{n}} \beta_{1}\left(y_{1}\right)=\min _{y_{1} \in K} \beta_{1}\left(y_{1}\right), \\
t_{0} & :=\min _{y_{1} \in \mathbb{R}^{n}} t_{1}\left(y_{1}\right)=\min _{y_{1} \in K} t_{1}\left(y_{1}\right) .
\end{aligned}
$$

Since $\tilde{y}(\cdot, \alpha, \beta, t)$ is weakly coercive, i. e.

$$
|\tilde{y}(x, \alpha, \beta, t)| \rightarrow \infty \quad \text { as } \quad|x| \rightarrow \infty,
$$

we get the existence of a global inverse of $\tilde{y}(\cdot, \alpha, \beta, t)$ if we restrict the set of definition of $\tilde{y}$ to $\mathbb{R}^{n} \times\left(-\alpha_{0}, \alpha_{0}\right) \times\left(-\beta_{0}, \beta_{0}\right) \times\left(-t_{0}, t_{0}\right)$. Hence $\tilde{y}(\cdot, \alpha, \beta, t)$ is also a $C^{\infty}$-diffeomorphism globally on $\mathbb{R}^{n}$ for any fixed $\alpha \in\left(-\alpha_{0}, \alpha_{0}\right), \beta \in\left(-\beta_{0}, \beta_{0}\right)$, $t \in\left(-t_{0}, t_{0}\right)$.

Now we show that the image of $\tilde{y}$ restricted to $\Omega \times\left(-\alpha_{0}, \alpha_{0}\right) \times\left(-\beta_{0}, \beta_{0}\right) \times\left(-t_{0}, t_{0}\right)$ lies in $\Omega$. For $x \in \mathbb{R}^{n} \backslash K$ we have $\tilde{y}(x, \alpha, \beta, t)=x$. Since $\tilde{y}(\cdot, \alpha, \beta, t)$ is injective on $\mathbb{R}^{n}$, we have $\tilde{y}(x, \alpha, \beta, t) \in K$ whenever $x \in K$. Therefore

$$
\tilde{y}\left(\Omega \times\left(-\alpha_{0}, \alpha_{0}\right) \times\left(-\beta_{0}, \beta_{0}\right) \times\left(-t_{0}, t_{0}\right)\right) \subset \Omega .
$$

The matrix $D \tilde{y}(x, \alpha, \beta, t)$ is regular for any

$$
(x, \alpha, \beta, t) \in \mathbb{R}^{n} \times\left(-\alpha_{0}, \alpha_{0}\right) \times\left(-\beta_{0}, \beta_{0}\right) \times\left(-t_{0}, t_{0}\right),
$$

since $\tilde{y}(\cdot, \alpha, \beta, t)$ is a $C^{\infty}$-diffeomorphism. Moreover,

$$
\operatorname{det} D \tilde{y}(x, \alpha, \beta, t)=1
$$

for any $x \in \mathbb{R}^{n} \backslash K$. Since $D \tilde{y}$ is regular we have $\operatorname{det} D \tilde{y}(x, \alpha, \beta, t)>0$ for any $(x, \alpha, \beta, t)$. Hence it must attain a positive minimum as a function of $x$ on the compact set $K$ and we get the assertion. 
As a simple consequence of Lemma 3.3 and its proof we state the following corollary.

Corollary 3.4. We denote the inverse of $\tilde{y}(\cdot, \alpha, \beta, t)$ by

$$
\tilde{x}: \Omega \times\left(-\alpha_{0}, \alpha_{0}\right) \times\left(-\beta_{0}, \beta_{0}\right) \times\left(-t_{0}, t_{0}\right) \rightarrow \Omega
$$

and its partial derivative with respect to $y$ by

$$
D \tilde{x}(y, \alpha, \beta, t):=\frac{\partial}{\partial y} \tilde{x}(y, \alpha, \beta, t)
$$

We have

$$
\tilde{x} \in C^{\infty}\left(\Omega \times\left(-\alpha_{0}, \alpha_{0}\right) \times\left(-\beta_{0}, \beta_{0}\right) \times\left(-t_{0}, t_{0}\right), \Omega\right)
$$

and $\tilde{x}(\cdot, \alpha, \beta, t)$ is a $C^{\infty}$-diffeomorphism on $\Omega$. Moreover, on $\Omega \backslash K$ we have

$$
\tilde{x}(y, \alpha, \beta, t)=y
$$

for all $\alpha, \beta, t$ and the compact set $K \subset \Omega$ as defined in (3.11).

Lemma 3.5. We have $0 \in \operatorname{int}_{Y}(D \cap Y)$ for every linear subspace $Y \subset X$ with $\operatorname{dim} Y \leq 2$.

Proof. Let $Y \subset X$ be a linear subspace of $X$ with $\operatorname{dim} Y \leq 2$ as defined in (3.10). It is $0 \in Y$ and $0 \in D$ and therefore $0 \in D \cap Y$.

We define a norm on $\mathbb{R}^{2}$ by

$$
\|(\alpha, \beta)\|_{Y}:=\left\|\alpha \eta_{1}+\beta \eta_{2}\right\|_{C^{1}}
$$

for fixed $\eta_{1}, \eta_{2}$ as in (3.10). Since all norms are equivalent on $\mathbb{R}^{2}$, it is sufficient to find a neighborhood $U$ of 0 in $\mathbb{R}^{2}$ with respect to the Euclidean norm, such that for all $(\alpha, \beta) \subset U$ we have $\alpha \eta_{1}+\beta \eta_{2} \in D$.

Lemma 3.3 states that there exist $\alpha_{0}>0, \beta_{0}>0$ such that for $t=0$

$$
\tilde{y}(x, \alpha, \beta, t)=x+\alpha \eta_{1}(x)+\beta \eta_{2}(x)
$$

is a $C^{\infty}$-diffeomorphism with respect to $x$ for all $x \in \Omega, \alpha \in\left(-\alpha_{0}, \alpha_{0}\right), \beta \in$ $\left(-\beta_{0}, \beta_{0}\right)$, i. e. $\alpha \eta_{1}+\beta \eta_{2} \in D$ and the assertion is satisfied. 
For the calculation of the directional derivatives $\delta \mathcal{E}(\eta, \xi), \delta \mathcal{G}(\eta, \xi)$ we need to know how $\tilde{x}$ and $\operatorname{det} D \tilde{x}$ depend on $t$.

Lemma 3.6. For any fixed $\xi \in X$ we define functions $\hat{x}: \Omega \times\left(-\alpha_{0}, \alpha_{0}\right) \times$ $\left(-\beta_{0}, \beta_{0}\right) \rightarrow \Omega$ and $\mathcal{Y}: \Omega \times\left(-\alpha_{0}, \alpha_{0}\right) \times\left(-\beta_{0}, \beta_{0}\right) \rightarrow \mathbb{R}^{n \times n}$ by

$$
\begin{gathered}
\hat{x}(y, \alpha, \beta):=\tilde{x}(y, \alpha, \beta, 0), \\
\mathcal{Y}(y, \alpha, \beta):=D \tilde{y}(\hat{x}(y, \alpha, \beta), \alpha, \beta, 0) .
\end{gathered}
$$

Then there exists a function $R: \Omega \times\left(-\alpha_{0}, \alpha_{0}\right) \times\left(-\beta_{0}, \beta_{0}\right) \times\left(-t_{0}, t_{0}\right) \rightarrow \mathbb{R}^{n}$ with $R(y, \alpha, \beta, t)=o(t)$ as $t \rightarrow 0$ for any fixed $y \in \Omega, \alpha \in\left(-\alpha_{0}, \alpha_{0}\right), \beta \in\left(-\beta_{0}, \beta_{0}\right)$, such that

$$
\tilde{x}(y, \alpha, \beta, t)=\hat{x}(y, \alpha, \beta)-t \mathcal{Y}(y, \alpha, \beta)^{-1} \xi(\hat{x}(y, \alpha, \beta))+R(y, \alpha, \beta, t) .
$$

Proof. By Corollary 3.4 we know that

$$
\tilde{x} \in C^{\infty}\left(\Omega \times\left(-\alpha_{0}, \alpha_{0}\right) \times\left(-\beta_{0}, \beta_{0}\right) \times\left(-t_{0}, t_{0}\right), \Omega\right)
$$

and thus we can expand $\tilde{x}$ in $t=0$ into

$$
\tilde{x}(y, \alpha, \beta, t)=\tilde{x}(y, \alpha, \beta, 0)+t \frac{\partial}{\partial t} \tilde{x}(y, \alpha, \beta, 0)+o(t)
$$

For the calculation of the derivative we use the implicit function theorem to get

$$
\begin{aligned}
\frac{\partial}{\partial t} \tilde{x}(y, \alpha, \beta, 0) & =-D \tilde{y}(\tilde{x}(y, \alpha, \beta, 0), \alpha, \beta, 0)^{-1} \xi(\tilde{x}(y, \alpha, \beta, 0)) \\
& =-\mathcal{Y}(y, \alpha, \beta)^{-1} \xi(\hat{x}(y, \alpha, \beta))
\end{aligned}
$$

We define

$$
R(y, \alpha, \beta, t):=\tilde{x}(y, \alpha, \beta, t)-\hat{x}(y, \alpha, \beta)+t \mathcal{Y}(y, \alpha, \beta)^{-1} \xi(\hat{x}(y, \alpha, \beta)) .
$$

By (3.13) we have $R(y, \alpha, \beta, t)=o(t)$ for any fixed $y \in \Omega, \alpha \in\left(-\alpha_{0}, \alpha_{0}\right), \beta \in$ $\left(-\beta_{0}, \beta_{0}\right)$. 
Lemma 3.7. For any fixed $\xi \in X$ we define $\mathcal{Q}: \Omega \times\left(-\alpha_{0}, \alpha_{0}\right) \times\left(-\beta_{0}, \beta_{0}\right) \rightarrow \mathbb{R}$ by

$$
\begin{aligned}
\mathcal{Q}(y, \alpha, \beta):=(\operatorname{det} \mathcal{Y}(y, \alpha, \beta))^{-1} & \\
\cdot \operatorname{tr}\left(\mathcal{Y}(y, \alpha, \beta)^{-1}(D \xi(\hat{x}(y, \alpha, \beta))-(\right. & \left.\alpha D^{2} \eta_{1}(\hat{x}(y, \alpha, \beta))+\beta D^{2} \eta_{2}(\hat{x}(y, \alpha, \beta))\right) \\
& \left.\left.\cdot \mathcal{Y}(y, \alpha, \beta)^{-1} \xi(\hat{x}(y, \alpha, \beta))\right)\right) .
\end{aligned}
$$

There exists a function $\mathcal{R}: \Omega \times\left(-\alpha_{0}, \alpha_{0}\right) \times\left(-\beta_{0}, \beta_{0}\right) \times\left(-t_{0}, t_{0}\right) \rightarrow \mathbb{R}$ with $\mathcal{R}(y, \alpha, \beta, t)=o(t)$ for any fixed $y \in \Omega, \alpha \in\left(-\alpha_{0}, \alpha_{0}\right), \beta \in\left(-\beta_{0}, \beta_{0}\right)$, such that

$$
\operatorname{det} D \tilde{x}(y, \alpha, \beta, t)=\operatorname{det} \mathcal{Y}(y, \alpha, \beta)^{-1}-t \mathcal{Q}(y, \alpha, \beta)+\mathcal{R}(y, \alpha, \beta, t) \text {. }
$$

Moreover, there exists a constant $c \in \mathbb{R}^{+}$with

$$
\operatorname{det} D \tilde{x}(x, \alpha, \beta, t) \geq c>0
$$

for any $(x, \alpha, \beta, t) \in \Omega \times\left(-\alpha_{0}, \alpha_{0}\right) \times\left(-\beta_{0}, \beta_{0}\right) \times\left(-t_{0}, t_{0}\right)$.

Proof. The function $\operatorname{det} D \tilde{x}(y, \alpha, \beta, \cdot)$ is differentiable, since $\tilde{x}$ is $C^{\infty}$ and the determinant is differentiable. Therefore we can expand it in $t=0$ into

$$
\operatorname{det} D \tilde{x}(y, \alpha, \beta, t)=\operatorname{det} D \tilde{x}(y, \alpha, \beta, 0)+t \frac{\partial}{\partial t} \operatorname{det} D \tilde{x}(y, \alpha, \beta, 0)+o(t)
$$

By the inverse function theorem we have

$$
D \tilde{x}(y, \alpha, \beta, t)=D \tilde{y}(\tilde{x}(y, \alpha, \beta, t), \alpha, \beta, t)^{-1}
$$

and therefore

$$
\begin{aligned}
\operatorname{det} D \tilde{x}(y, \alpha, \beta, 0) & =\operatorname{det} D \tilde{y}(\hat{x}(y, \alpha, \beta), \alpha, \beta, 0)^{-1} \\
& =\operatorname{det} \mathcal{Y}(y, \alpha, \beta)^{-1}
\end{aligned}
$$

In the following calculation we omit the variables $\alpha, \beta$ as $\operatorname{argument}$ of $\tilde{x}, \tilde{y}, \hat{x}$ and $\mathcal{Y}$ for short. We use a differentiation rule for determinants, cf. Bernstein $[5$, 
10.8.11], and calculate

$$
\begin{aligned}
& \left.\frac{\partial}{\partial t} \operatorname{det} D \tilde{x}(y, t)\right|_{t=0}=\left.\frac{\partial}{\partial t}(\operatorname{det} D \tilde{y}(\tilde{x}(y, t), t))^{-1}\right|_{t=0} \\
& =-\left.\frac{\frac{\partial}{\partial t} \operatorname{det} D \tilde{y}(\tilde{x}(y, t), t)}{(\operatorname{det} D \tilde{y}(\tilde{x}(y, t), t))^{2}}\right|_{t=0} \\
& =-\frac{\operatorname{tr}\left(\left.\operatorname{adj} D \tilde{y}(\hat{x}(y), 0) \frac{\partial}{\partial t} D \tilde{y}(\tilde{x}(y, t), t)\right|_{t=0}\right)}{(\operatorname{det} D \tilde{y}(\hat{x}(y), 0))^{2}} \\
& =-\frac{\operatorname{tr}\left(\left.\operatorname{adj} \mathcal{Y}(y) \frac{\partial}{\partial t}\left(\operatorname{id}+\alpha D \eta_{1}(\tilde{x}(y, t))+\beta D \eta_{2}(\tilde{x}(y, t))+t D \xi(\tilde{x}(y, t))\right)\right|_{t=0}\right)}{(\operatorname{det} \mathcal{Y}(y))^{2}} \\
& =-\frac{\operatorname{tr}\left(\operatorname{adj} \mathcal{Y}(y)\left(\left(\alpha D^{2} \eta_{1}(\hat{x}(y))+\beta D^{2} \eta_{2}(\hat{x}(y))\right) \frac{\partial}{\partial t} \tilde{x}(y, 0)+D \xi(\hat{x}(y))\right)\right)}{(\operatorname{det} \mathcal{Y}(y))^{2}} \\
& \stackrel{(3.14)}{=}-\frac{\operatorname{tr}\left(\operatorname{adj} \mathcal{Y}(y)\left(D \xi(\hat{x}(y))-\left(\alpha D^{2} \eta_{1}(\hat{x}(y))+\beta D^{2} \eta_{2}(\hat{x}(y))\right) \mathcal{Y}(y)^{-1} \xi(\hat{x}(y))\right)\right)}{(\operatorname{det} \mathcal{Y}(y))^{2}} .
\end{aligned}
$$

We use that $\operatorname{adj} \mathcal{Y}(y)=\mathcal{Y}(y)^{-1} \operatorname{det} \mathcal{Y}(y)$ and the linearity of the trace to get

$$
\begin{aligned}
& \left.\frac{\partial}{\partial t} \operatorname{det} D \tilde{x}(y, t)\right|_{t=0} \\
& =-\frac{\operatorname{tr}\left(\mathcal{Y}(y)^{-1}\left(D \xi(\hat{x}(y))-\left(\alpha D^{2} \eta_{1}(\hat{x}(y))+\beta D^{2} \eta_{2}(\hat{x}(y))\right) \mathcal{Y}(y)^{-1} \xi(\hat{x}(y))\right)\right)}{\operatorname{det} \mathcal{Y}(y)} \\
& =-\mathcal{Q}(y)
\end{aligned}
$$

Let us define

$$
\mathcal{R}(y, \alpha, \beta, t):=\operatorname{det} D \tilde{x}(y, \alpha, \beta, t)-\operatorname{det} \mathcal{Y}(y, \alpha, \beta)^{-1}+t \mathcal{Q}(y, \alpha, \beta)
$$

Then $\mathcal{R}(y, \alpha, \beta, t)=o(t)$ by definition for any fixed $y \in \Omega, \alpha \in\left(-\alpha_{0}, \alpha_{0}\right)$, $\beta \in\left(-\beta_{0}, \beta_{0}\right)$.

We have

$$
\operatorname{det} D \tilde{x}(y, \alpha, \beta, t)=(\operatorname{det} D \tilde{y}(\tilde{x}(y, \alpha, \beta, t), \alpha, \beta, t))^{-1}>0
$$

for any $(y, \alpha, \beta, t)$ by Lemma 3.3. For any $y \in \Omega \backslash K$, with $K$ as defined in (3.11), 
we have $\operatorname{det} D \tilde{x}(y, \alpha, \beta, t)=1$, cf. Corollary 3.4. Hence a positive minimum of $\operatorname{det} D \tilde{x}(\cdot, \alpha, \beta, t)$ must be attained on the compact set $K$ and we get the assertion.

Now we show that $u(\tilde{y}(\cdot, \alpha, \beta, t))$ has bounded variation on $\Omega$ and determine how its total variation can be represented in terms of $|D u|, \tilde{y}, \tilde{x}$.

Lemma 3.8. Let $u \in B V(\Omega), \eta \in D \cap Y$, cf. (3.7), (3.10), and $v: \Omega \rightarrow \mathbb{R}$ with

$$
v(x):=u(\tilde{y}(x, \alpha, \beta, t))
$$

for any fixed $\alpha \in\left(-\alpha_{0}, \alpha_{0}\right), \beta \in\left(-\beta_{0}, \beta_{0}\right), t \in\left(-t_{0}, t_{0}\right)$. Then $v \in B V(\Omega)$ and $v \equiv u$ in the sense of trace on $\partial \Omega$ for any fixed $\alpha \in\left(-\alpha_{0}, \alpha_{0}\right), \beta \in\left(-\beta_{0}, \beta_{0}\right)$, $t \in\left(-t_{0}, t_{0}\right)$. Let $z: \Omega \rightarrow \mathbb{R}^{n}$ be a vector field according to (3.4). Then

$$
\int_{\Omega}|v(x)| d x=\int_{\Omega}|u(y)| \operatorname{det} D \tilde{x}(y, \alpha, \beta, t) d y
$$

and

$$
\int_{\Omega} d|D v|=\int_{\Omega}\left|D \tilde{y}(\tilde{x}(y, \alpha, \beta, t), \alpha, \beta, t)^{T} z(y)\right| \operatorname{det} D \tilde{x}(y, \alpha, \beta, t) d|D u| .
$$

For the proof of Lemma 3.8 we need the following Proposition, cf. Ciarlet [11, Theorem 1.7-1] for the case $n=3$.

Proposition 3.9. (Properties of the Piola transform)

Let $\Omega, \tilde{\Omega} \subset \mathbb{R}^{n}$ be open, $\varphi \in C^{2}(\Omega, \tilde{\Omega})$ with $D \varphi(x)$ regular for all $x \in \Omega$, and $\psi \in C^{1}\left(\tilde{\Omega}, \mathbb{R}^{n}\right)$. Let $\psi^{*}: \Omega \rightarrow \mathbb{R}^{n}$ be defined as

$$
\psi^{*}(x):=D \varphi(x)^{-1} \psi(\varphi(x)) \operatorname{det} D \varphi(x) .
$$

Then

$$
\operatorname{div} \psi^{*}(x)=\operatorname{div} \psi(\varphi(x)) \operatorname{det} D \varphi(x) .
$$

Proof. The key to the proof is the Piola identity

$$
\operatorname{div}\left(D \varphi(x)^{-T} \operatorname{det} D \varphi(x)\right)=0,
$$


cf. Zeidler $[40$, p. $548,12.2 \mathrm{~b}]$. Note that $\operatorname{div}\left(D \varphi(x)^{-T} \operatorname{det} D \varphi(x)\right)$ is a vector whose components are the divergences of the row vectors in the matrix $D \varphi(x)^{-T}$ det $D \varphi(x)$. More explicitly, we have for the $j$-th component

$$
\begin{aligned}
\left(\operatorname{div}\left(D \varphi(x)^{-T} \operatorname{det} D \varphi(x)\right)\right)_{j} & =\sum_{i=1}^{n} \frac{\partial}{\partial x_{i}}\left(D \varphi(x)_{j i}^{-T} \operatorname{det} D \varphi(x)\right) \\
& =\sum_{i=1}^{n} \frac{\partial}{\partial x_{i}}\left(D \varphi(x)_{i j}^{-1} \operatorname{det} D \varphi(x)\right)=0
\end{aligned}
$$

for $j=1, \ldots, n$. Thus for

$$
\psi_{i}^{*}(x)=\sum_{j=1}^{n} D \varphi(x)_{i j}^{-1} \psi_{j}(\varphi(x)) \operatorname{det} D \varphi(x)
$$

for $i=1, \ldots, n$ we have

$$
\begin{aligned}
\operatorname{div} \psi^{*}(x) & =\sum_{i=1}^{n} \frac{\partial}{\partial x_{i}} \psi_{i}^{*}(x) \\
= & \sum_{i=1}^{n} \frac{\partial}{\partial x_{i}} \sum_{j=1}^{n} D \varphi(x)_{i j}^{-1} \psi_{j}(\varphi(x)) \operatorname{det} D \varphi(x) \\
= & \sum_{j=1}^{n} \sum_{i=1}^{n} \frac{\partial}{\partial x_{i}}\left(D \varphi(x)_{i j}^{-1} \operatorname{det} D \varphi(x)\right) \psi_{j}(\varphi(x)) \\
& \quad+\sum_{i=1}^{n} \sum_{j=1}^{n} D \varphi(x)_{i j}^{-1} \operatorname{det} D \varphi(x) \frac{\partial}{\partial x_{i}} \psi_{j} \circ \varphi(x),
\end{aligned}
$$

while we applied the product rule in the last line. The sum in (3.21) equals zero by (3.20). For the calculation of (3.22) we apply the chain rule

$$
\frac{\partial}{\partial x_{i}} \psi_{j} \circ \varphi(x)=\sum_{k=1}^{n} \frac{\partial}{\partial x_{k}} \psi_{j}(\varphi(x)) \frac{\partial}{\partial x_{i}} \varphi_{k}(x)=\sum_{k=1}^{n} \frac{\partial}{\partial x_{k}} \psi_{j}(\varphi(x)) D \varphi(x)_{k i} .
$$

Thus we get

$$
\operatorname{div} \psi^{*}(x)=\sum_{i=1}^{n} \sum_{j=1}^{n} D \varphi(x)_{i j}^{-1} \operatorname{det} D \varphi(x) \frac{\partial}{\partial x_{i}} \psi_{j} \circ \varphi(x)
$$




$$
\begin{aligned}
& =\sum_{j=1}^{n} \sum_{k=1}^{n}(\underbrace{\sum_{i=1}^{n} D \varphi(x)_{k i} D \varphi(x)_{i j}^{-1}}_{=\delta_{j k}}) \operatorname{det} D \varphi(x) \frac{\partial}{\partial x_{k}} \psi_{j}(\varphi(x)) \\
& =\sum_{j=1}^{n} \operatorname{det} D \varphi(x) \frac{\partial}{\partial x_{j}} \psi_{j}(\varphi(x)) \\
& =\operatorname{div} \psi(\varphi(x)) \operatorname{det} D \varphi(x) .
\end{aligned}
$$

Proof of Lemma 3.8. We have $u \in L^{1}(\Omega)$, $\operatorname{det} D \tilde{x}(y, \alpha, \beta, t)>0$ for all $(y, \alpha, \beta, t)$ by Lemma 3.7. Therefore

$$
\int_{\Omega}|v(x)| d x=\int_{\Omega}|u(\tilde{y}(x, \alpha, \beta, t))| d x=\int_{\Omega}|u(y)| \operatorname{det} D \tilde{x}(y, \alpha, \beta, t) d y .
$$

We consider an arbitrary vector field $\psi \in C_{0}^{1}\left(\Omega, \mathbb{R}^{n}\right)$ with $\|\psi\|_{\infty} \leq 1$ and define $\psi^{*}: \Omega \rightarrow \mathbb{R}^{n}$ by

$$
\psi^{*}(y):=D \tilde{x}(y, \alpha, \beta, t)^{-1} \psi(\tilde{x}(y, \alpha, \beta, t)) \operatorname{det} D \tilde{x}(y, \alpha, \beta, t)
$$

for fixed $\alpha, \beta$, t. Since $\psi \in C_{0}^{1}\left(\Omega, \mathbb{R}^{n}\right)$ and all other functions involved $C^{\infty}$, we get also $\psi^{*} \in C_{0}^{1}\left(\Omega, \mathbb{R}^{n}\right)$. So we calculate

$$
\begin{aligned}
& \int_{\Omega} v(x) \operatorname{div} \psi(x) d x=\int_{\Omega} u(\tilde{y}(x, \alpha, \beta, t)) \operatorname{div} \psi(x) d x \\
& =\int_{\Omega} u(y) \operatorname{div} \psi(\tilde{x}(y, \alpha, \beta, t)) \operatorname{det} D \tilde{x}(y, \alpha, \beta, t) d y \\
& \stackrel{(3.19)}{=} \int_{\Omega} u(y) \operatorname{div} \psi^{*}(y) d y \\
& =-\int_{\Omega} \psi^{*}(y) d(D u) \\
& =-\int_{\Omega} D \tilde{x}(y, \alpha, \beta, t)^{-1} \psi(\tilde{x}(y, \alpha, \beta, t)) \operatorname{det} D \tilde{x}(y, \alpha, \beta, t) d(D u) \\
& =-\int_{\Omega}\left\langle D \tilde{x}(y, \alpha, \beta, t)^{-1} \psi(\tilde{x}(y, \alpha, \beta, t)), z(y)\right\rangle \operatorname{det} D \tilde{x}(y, \alpha, \beta, t) d|D u| \\
& =-\int_{\Omega}\left\langle\psi(\tilde{x}(y, \alpha, \beta, t)), D \tilde{x}(y, \alpha, \beta, t)^{-T} z(y)\right\rangle \operatorname{det} D \tilde{x}(y, \alpha, \beta, t) d|D u| .
\end{aligned}
$$


We define the $f: \Omega \rightarrow \mathbb{R}^{n}$ by

$$
f(y):=D \tilde{x}(y, \alpha, \beta, t)^{-T} z(y) \operatorname{det} D \tilde{x}(y, \alpha, \beta, t)
$$

for fixed $\alpha, \beta, t$ and consider the $\mathbb{R}^{n}$-valued Radon measure $f|D u|$ on $\Omega$, cf. Ambrosio et al. [2, Definition 1.22]. Note that $f$ is indeed in $L^{1}\left(\Omega, \mathbb{R}^{n}\right)$ since $D \tilde{x}(y, \alpha, \beta, t)^{-T}=D \tilde{y}(\tilde{x}(y, \alpha, \beta, t), \alpha, \beta, t)^{T}$ is regular and $z \in L^{1}\left(\Omega, \mathbb{R}^{n}\right)$. By $[2$, Proposition 1.23] the total variation of the vector measure $f|D u|$ is given by

$$
|f| D u||(\Omega)=\int_{\Omega}|f| d|D u|
$$

Moreover, by definition of the total variation we have

$$
\begin{aligned}
|f| D u||(\Omega) & =\sup \left\{\int_{\Omega} \psi d(f|D u|) \mid \psi \in C_{0}\left(\Omega, \mathbb{R}^{n}\right),\|\psi\|_{\infty} \leq 1\right\} \\
& =\sup \left\{\int_{\Omega}\langle\psi, f\rangle d|D u| \mid \psi \in C_{0}\left(\Omega, \mathbb{R}^{n}\right),\|\psi\|_{\infty} \leq 1\right\}
\end{aligned}
$$

cf. [2, Proposition 1.47]. For any $\psi \in C_{0}\left(\Omega, \mathbb{R}^{n}\right)$ we can always find a sequence $\left(\psi_{k}\right)_{k \in \mathbb{N}}$ with $\psi_{k} \in C_{0}^{1}\left(\Omega, \mathbb{R}^{n}\right)$ such that $\left\|\psi-\psi_{k}\right\|_{\infty} \rightarrow 0$ as $k \rightarrow \infty$. But then we also have

$$
\begin{aligned}
\left|\int_{\Omega}\langle\psi, f\rangle d\right| D u \mid & -\int_{\Omega}\left\langle\psi_{k}, f\right\rangle d|D u||=| \int_{\Omega}\left\langle\psi-\psi_{k}, f\right\rangle d|D u| \mid \\
& \leq\left\|\psi-\psi_{k}\right\|_{\infty} \int_{\Omega}|f| d|D u| \rightarrow 0
\end{aligned}
$$

for $k \rightarrow \infty$. Therefore we can also take the supremum over $\psi \in C_{0}^{1}\left(\Omega, \mathbb{R}^{n}\right)$ with $\|\psi\|_{\infty} \leq 1$ in (3.26). Moreover, given any $\hat{\psi} \in C_{0}^{1}\left(\Omega, \mathbb{R}^{n}\right)$ with $\|\hat{\psi}\|_{\infty} \leq 1$ we can define a function $\psi \in C_{0}^{1}\left(\Omega, \mathbb{R}^{n}\right)$ by $\psi(x):=\hat{\psi}(\tilde{y}(x, \alpha, \beta, t))$ for fixed $\alpha, \beta, t$. Then, since $\tilde{x}(\cdot, \alpha, \beta, t)$ is bijective in $\Omega$, we have

$$
\psi(\tilde{x}(y, \alpha, \beta, t))=\hat{\psi}(\tilde{y}(\tilde{x}(y, \alpha, \beta, t), \alpha, \beta, t))=\hat{\psi}(y)
$$

Therefore we can conclude

$$
\sup _{\psi \in C_{0}^{1},\|\psi\|_{\infty} \leq 1} \int_{\Omega} v(x) \operatorname{div} \psi(x) d x
$$




$$
\begin{aligned}
& \stackrel{(3.24)}{=} \sup _{\psi \in C_{0}^{1},\|\psi\|_{\infty} \leq 1} \int_{\Omega}\left\langle\psi(\tilde{x}(y, \alpha, \beta, t)), D \tilde{x}(y, \alpha, \beta, t)^{-T} z(y)\right\rangle \operatorname{det} D \tilde{x}(y, \alpha, \beta, t) d|D u| \\
& =\sup _{\hat{\psi} \in C_{0}^{1},\|\hat{\psi}\|_{\infty} \leq 1} \int_{\Omega}\left\langle\hat{\psi}(y), D \tilde{x}(y, \alpha, \beta, t)^{-T} z(y)\right\rangle \operatorname{det} D \tilde{x}(y, \alpha, \beta, t) d|D u| \\
& \stackrel{(3.26)}{=}|f| D u \| \mid(\Omega) \\
& \stackrel{(3.25)}{=} \int_{\Omega}|f(y)| d|D u| \\
& =\int_{\Omega}\left|D \tilde{x}(y, \alpha, \beta, t)^{-T} z(y)\right| \operatorname{det} D \tilde{x}(y, \alpha, \beta, t) d|D u| \\
& =\int_{\Omega}\left|D \tilde{y}(\tilde{x}(y, \alpha, \beta, t), \alpha, \beta, t)^{T} z(y)\right| \operatorname{det} D \tilde{x}(y, \alpha, \beta, t) d|D u| .
\end{aligned}
$$

Since the right hand side is finite, we get $v \in B V(\Omega)$ and the assertion for $\int_{\Omega} d|D v|$ follows.

Since there exists a compact set $K$ such that we have $\tilde{y}(x, \alpha, \beta, t)=x$ for each $x \in \Omega \backslash K$, cf. (3.11), we get $v \equiv u$ on $\partial \Omega$ in the sense of trace.

Now the functionals $\mathcal{E}$ and $\mathcal{G}$ as defined in (3.8), (3.9) are well defined on $D$.

Lemma 3.10. The directional derivatives $\delta \mathcal{E}(\cdot, \xi), \delta \mathcal{G}(\cdot, \xi): D \cap Y \rightarrow \mathbb{R}$ are

$$
\begin{aligned}
\delta \mathcal{E}(\eta, \xi)= & \int_{\Omega}\left\langle\left( D \xi(\hat{x}(y, \alpha, \beta))-\left(\alpha D^{2} \eta_{1}(\hat{x}(y, \alpha, \beta))+\beta D^{2} \eta_{2}(\hat{x}(y, \alpha, \beta))\right)\right.\right. \\
& \left.\left.\cdot \mathcal{Y}(y, \alpha, \beta)^{-1} \xi(\hat{x}(y, \alpha, \beta))\right)^{T} z(y), \frac{\mathcal{Y}(y, \alpha, \beta)^{T} z(y)}{\left|\mathcal{Y}(y, \alpha, \beta)^{T} z(y)\right|}\right\rangle \operatorname{det} \mathcal{Y}(y, \alpha, \beta)^{-1} \\
& -\left|\mathcal{Y}(y, \alpha, \beta)^{T} z(y)\right| \mathcal{Q}(y, \alpha, \beta) d|D u|
\end{aligned}
$$

and

$$
\delta \mathcal{G}(\eta, \xi)=-\int_{\Omega}|u(y)| \mathcal{Q}(y, \alpha, \beta) d y
$$

for any fixed $\xi \in X$ and $\eta=\alpha \eta_{1}+\beta \eta_{2}$.

Proof. We use Lemma 3.7 and Lemma 3.8 and get for $v$ according to (3.18)

$$
\begin{aligned}
\mathcal{E}( & +t \xi)=\int_{\Omega} d|D v| \\
& =\int_{\Omega}\left|D \tilde{y}(\tilde{x}(y, \alpha, \beta, t), \alpha, \beta, t)^{T} z(y)\right| \operatorname{det} D \tilde{x}(y, \alpha, \beta, t) d|D u|
\end{aligned}
$$




$$
\begin{aligned}
& =\int_{\Omega}\left|D \tilde{y}(\tilde{x}(y, \alpha, \beta, t), \alpha, \beta, t)^{T} z(y)\right| \\
& \quad \cdot\left(\operatorname{det} \mathcal{Y}(y, \alpha, \beta)^{-1}-t \mathcal{Q}(y, \alpha, \beta)+\mathcal{R}(y, \alpha, \beta, t)\right) d|D u| \\
& =\int_{\Omega}\left|D \tilde{y}(\tilde{x}(y, \alpha, \beta, t), \alpha, \beta, t)^{T} z(y)\right| \operatorname{det} \mathcal{Y}(y, \alpha, \beta)^{-1} d|D u| \\
& \quad-\int_{\Omega} t\left|D \tilde{y}(\tilde{x}(y, \alpha, \beta, t), \alpha, \beta, t)^{T} z(y)\right| \mathcal{Q}(y, \alpha, \beta) d|D u| \\
& \quad+\int_{\Omega}\left|D \tilde{y}(\tilde{x}(y, \alpha, \beta, t), \alpha, \beta, t)^{T} z(y)\right| \mathcal{R}(y, \alpha, \beta, t) d|D u| .
\end{aligned}
$$

Now we calculate the directional derivative

$$
\delta \mathcal{E}(\eta, \xi)=\left.\frac{\partial}{\partial t} \int_{\Omega} d|D v|\right|_{t=0}
$$

separately for the terms (3.27), (3.28), (3.29). We use a result on the differentiability of parameter-dependent integrals as it can be found in Schilling [38, Theorem 11.5].

We omit $\alpha$ and $\beta$ as arguments in $\tilde{x}, \tilde{y}, \hat{x}, \mathcal{Y}, \mathcal{Q}$ for simplicity and calculate the derivatives of the integrands. First of all we get

$$
\begin{aligned}
& \mathcal{F}(y, t):=\frac{\partial}{\partial t}\left|D \tilde{y}(\tilde{x}(y, t), t)^{T} z(y)\right| \\
&=\left\langle\frac{D \tilde{y}(\tilde{x}(y, t), t)^{T} z(y)}{\left|D \tilde{y}(\tilde{x}(y, t), t)^{T} z(y)\right|}, \frac{\partial}{\partial t} D \tilde{y}(\tilde{x}(y, t), t)^{T} z(y)\right\rangle \\
&=\left\langle\frac{D \tilde{y}(\tilde{x}(y, t), t)^{T} z(y)}{\left|D \tilde{y}(\tilde{x}(y, t), t)^{T} z(y)\right|},\right. \\
&\left.\frac{\partial}{\partial t}\left(\mathrm{id}+\alpha D \eta_{1}(\tilde{x}(y, t))+\beta D \eta_{2}(\tilde{x}(y, t))+t D \xi(\tilde{x}(y, t))\right)^{T} z(y)\right\rangle \\
&=\left\langle\frac{D \tilde{y}(\tilde{x}(y, t), t)^{T} z(y)}{\left|D \tilde{y}(\tilde{x}(y, t), t)^{T} z(y)\right|},\left(\left(\alpha D^{2} \eta_{1}(\tilde{x}(y, t))+\beta D^{2} \eta_{2}(\tilde{x}(y, t))\right)\right.\right. \\
&\left.\left.\quad \cdot \frac{\partial}{\partial t} \tilde{x}(y, t)+D \xi(\tilde{x}(y, t))+t D^{2} \xi(\tilde{x}(y, t)) \frac{\partial}{\partial t} \tilde{x}(y, t)\right)^{T} z(y)\right\rangle .
\end{aligned}
$$

Thus for the first integrand, cf. (3.27), we obtain

$$
\frac{\partial}{\partial t}\left|D \tilde{y}(\tilde{x}(y, t), t)^{T} z(y)\right| \operatorname{det} \mathcal{Y}(y)^{-1}=\mathcal{F}(y, t) \operatorname{det} \mathcal{Y}(y)^{-1},
$$


and for the second, cf. (3.28),

$$
\frac{\partial}{\partial t}\left(t\left|D \tilde{y}(\tilde{x}(y, t), t)^{T} z(y)\right|\right) \mathcal{Q}(y)=\left|D \tilde{y}(\tilde{x}(y, t), t)^{T} z(y)\right| \mathcal{Q}(y)+t \mathcal{F}(y, t) \mathcal{Q}(y)
$$

Using definition (3.17) of $\mathcal{R}$ we get for the third part, cf. (3.29),

$$
\begin{aligned}
\frac{\partial}{\partial t}\left(\left|D \tilde{y}(\tilde{x}(y, t), t)^{T} z(y)\right| \mathcal{R}(y, t)\right) \\
=\mathcal{F}(y, t) \mathcal{R}(y, t)+\left|D \tilde{y}(\tilde{x}(y, t), t)^{T} z(y)\right| \frac{\partial}{\partial t} \mathcal{R}(y, t) \\
=\mathcal{F}(y, t)\left(\operatorname{det} D \tilde{x}(y, t)-\operatorname{det} \mathcal{Y}(y)^{-1}+t \mathcal{Q}(y)\right) \\
\quad+\left|D \tilde{y}(\tilde{x}(y, t), t)^{T} z(y)\right|\left(\frac{\partial}{\partial t} \operatorname{det} D \tilde{x}(y, t)+\mathcal{Q}(y)\right) .
\end{aligned}
$$

Let us define the compact set

$$
M:=\bar{\Omega} \times\left[-\alpha_{0} / 2, \alpha_{0} / 2\right] \times\left[-\beta_{0} / 2, \beta_{0} / 2\right] \times\left[-t_{0} / 2, t_{0} / 2\right]
$$

We show that the calculated derivatives (3.31), (3.32), (3.33) are bounded by a constant for $|D u|$-a. e. $y$ and all $\alpha, \beta, t$ with $(y, \alpha, \beta, t) \in M$. Note that we do not require the boundedness in $\alpha, \beta$ here, but we will need it in the proof of the next Lemma. By looking closely at (3.31), (3.32), (3.33), we see that most of the terms involved are continuous on the compact set $M$ and therefore also bounded there. Thus we check only terms for which the continuity is not immediately clear.

Let us first of all state, that since we have $\tilde{x}(y, t)=y$ on $(\bar{\Omega} \backslash K) \times\left[-\alpha_{0} / 2, \alpha_{0} / 2\right] \times$ $\left[-\beta_{0} / 2, \beta_{0} / 2\right] \times\left[-t_{0} / 2, t_{0} / 2\right]$ by Corollary 3.4 , we get

$$
\frac{\partial}{\partial t} \tilde{x}(y, t)=0, \quad \operatorname{det} D \tilde{x}(y, t)=1, \quad \text { and } \quad \frac{\partial}{\partial t} \operatorname{det} D \tilde{x}(y, t)=0
$$

there with $K$ as defined in (3.11). Therefore the continuous functions $\frac{\partial}{\partial t} \tilde{x}$, det $D \tilde{x}$, $\frac{\partial}{\partial t} \operatorname{det} D \tilde{x}$ are bounded on the compact set $M$.

Thus, in (3.30) the only term to be checked for boundedness is

$$
\frac{D \tilde{y}(\tilde{x}(y, t), t)^{T} z(y)}{\left|D \tilde{y}(\tilde{x}(y, t), t)^{T} z(y)\right|} .
$$


By Lemma $3.3 D \tilde{y}$ is always a regular matrix and since $z(y) \neq 0|D u|$-a. e. on $\Omega$ we get

$$
\left|D \tilde{y}(\tilde{x}(y, t), t)^{T} z(y)\right|>0
$$

there. Therefore we have

$$
\left|\frac{D \tilde{y}(\tilde{x}(y, t), t)^{T} z(y)}{\left|D \tilde{y}(\tilde{x}(y, t), t)^{T} z(y)\right|}\right|=1
$$

for $|D u|$-a.e. $y$ and all $\alpha, \beta, t$ with $(y, \alpha, \beta, t) \in M$ and hence $\mathcal{F}$ as defined in (3.30) is bounded there.

In (3.31) we must only check the boundedness of

$$
(\operatorname{det} \mathcal{Y}(y))^{-1}=\operatorname{det} \mathcal{Y}(y)^{-1}=(\operatorname{det} D \tilde{y}(\tilde{x}(y, 0), 0))^{-1}
$$

But we already have $\operatorname{det} D \tilde{y}(\tilde{x}(y, 0), 0) \geq c>0$ for some $c \in \mathbb{R}^{+}$and all $(y, \alpha, \beta) \in$ $\bar{\Omega} \times\left[-\alpha_{0} / 2, \alpha_{0} / 2\right] \times\left[-\beta_{0} / 2, \beta_{0} / 2\right]$ by Lemma 3.3. Moreover, it follows that also

$$
\mathcal{Y}(y)^{-1}=(\operatorname{det} \mathcal{Y}(y))^{-1} \operatorname{adj} \mathcal{Y}(y)
$$

is bounded there, since each entry of adj $\mathcal{Y}(y)$ is bounded. This gives us the boundedness of (3.31) on $M$ and, using the definition of $\mathcal{Q}$, cf. Lemma 3.7, we see that the boundedness of $\mathcal{Q}$ follows, i. e. also (3.32) is bounded on $M$.

Now, using (3.34), the boundedness of (3.33) on $M$ is a simple consequence and in particular also

$$
\frac{\partial}{\partial t} \mathcal{R}(y, t)=\frac{\partial}{\partial t} \operatorname{det} D \tilde{x}(y, t)+\mathcal{Q}(y)
$$

is bounded on $M$.

Thus we may use [38, Theorem 11.5] and get

$$
\begin{aligned}
& \left.\frac{\partial}{\partial t} \int_{\Omega}\left|D \tilde{y}(\tilde{x}(y, t), t)^{T} z(y)\right| \operatorname{det} D \tilde{x}(y, t) d|D u|\right|_{t=0} \\
& \quad=\left.\int_{\Omega} \frac{\partial}{\partial t}\left(\left|D \tilde{y}(\tilde{x}(y, t), t)^{T} z(y)\right| \operatorname{det} D \tilde{x}(y, t)\right)\right|_{t=0} d|D u|
\end{aligned}
$$

In the case $t=0$ for the derivative of the first integrand as in (3.31) we have

$$
\left.\frac{\partial}{\partial t}\left|D \tilde{y}(\tilde{x}(y, t), t)^{T} z(y)\right| \operatorname{det} \mathcal{Y}(y)^{-1}\right|_{t=0}=\mathcal{F}(y, 0) \operatorname{det} \mathcal{Y}(y)^{-1}
$$


and for the derivative of the second integrand, cf. (3.32),

$$
\left.\frac{\partial}{\partial t}\left(t\left|D \tilde{y}(\tilde{x}(y, t), t)^{T} z(y)\right|\right) \mathcal{Q}(y)\right|_{t=0}=\left|\mathcal{Y}(y)^{T} z(y)\right| \mathcal{Q}(y)
$$

In (3.33) we get

$$
\left.\frac{\partial}{\partial t}\left|D \tilde{y}(\tilde{x}(y, t), t)^{T} z(y)\right| \mathcal{R}(y, t)\right|_{t=0}=0
$$

since $\operatorname{det} D \tilde{x}(y, 0)=\operatorname{det} \mathcal{Y}(y)^{-1}$ by $(3.15)$ and

$$
\left.\frac{\partial}{\partial t} \mathcal{R}(y, t)\right|_{t=0}=\left.\frac{\partial}{\partial t} \operatorname{det} D \tilde{x}(y, t)\right|_{t=0}+\mathcal{Q}(y)=0
$$

by (3.16). Moreover, using Lemma 3.3 we get

$$
\begin{aligned}
& \mathcal{F}(y, 0)=\left\langle\frac{\mathcal{Y}(y)^{T} z(y)}{\left|\mathcal{Y}(y)^{T} z(y)\right|}\right. \\
& \left.\quad\left(D \xi(\hat{x}(y))-\left(\alpha D^{2} \eta_{1}(\hat{x}(y))+\beta D^{2} \eta_{2}(\hat{x}(y))\right) \mathcal{Y}(y)^{-1} \xi(\hat{x}(y))\right)^{T} z(y)\right\rangle .
\end{aligned}
$$

Therefore, using (3.36), (3.37), (3.38), and (3.40), we obtain

$$
\begin{array}{r}
\delta \mathcal{E}(\eta, \xi)=\int_{\Omega}\left\langle\left(D \xi(\hat{x}(y))-\left(\alpha D^{2} \eta_{1}(\hat{x}(y))+\beta D^{2} \eta_{2}(\hat{x}(y))\right) \mathcal{Y}(y)^{-1} \xi(\hat{x}(y))\right)^{T} z(y),\right. \\
\left.\frac{\mathcal{Y}(y)^{T} z(y)}{\left|\mathcal{Y}(y)^{T} z(y)\right|}\right\rangle \operatorname{det} \mathcal{Y}(y)^{-1}-\left|\mathcal{Y}(y)^{T} z(y)\right| \mathcal{Q}(y) d|D u| .
\end{array}
$$

Now we look at

$$
\delta \mathcal{G}(\eta, \xi)=\left.\frac{\partial}{\partial t} \int_{\Omega}|v(x)| d x\right|_{t=0}
$$

We know by (3.23) that

$$
\mathcal{G}(\eta+t \xi)=\int_{\Omega}|v(x)| d x=\int_{\Omega}|u(y)| \operatorname{det} D \tilde{x}(y, \alpha, \beta, t) d y
$$

Using Lemma 3.7 we calculate the derivative of the integrand

$$
\begin{aligned}
\frac{\partial}{\partial t}(|u(y)| \operatorname{det} D \tilde{x}(y, t)) & =\frac{\partial}{\partial t}\left(|u(y)|\left(\operatorname{det} \mathcal{Y}(y)^{-1}-t \mathcal{Q}(y)+\mathcal{R}(y, t)\right)\right) \\
& =-|u(y)| \mathcal{Q}(y)+|u(y)| \frac{\partial}{\partial t} \mathcal{R}(y, t)
\end{aligned}
$$


We have already seen that $\mathcal{Q}$ is bounded on the compact set $\bar{\Omega} \times\left[-\alpha_{0} / 2, \alpha_{0} / 2\right] \times$ $\left[-\beta_{0} / 2, \beta_{0} / 2\right]$ and $\frac{\partial}{\partial t} \mathcal{R}$ on $M$, cf. (3.35). Using (3.39) we thus get

$$
\left.\frac{\partial}{\partial t}(|u(y)| \operatorname{det} D \tilde{x}(y, t))\right|_{t=0}=-|u(y)| \mathcal{Q}(y) .
$$

Now we use [38, Theorem 11.5] again and obtain

$$
\delta \mathcal{G}(\eta, \xi)=-\int_{\Omega}|u(y)| \mathcal{Q}(y) d y
$$

Lemma 3.11. The directional derivatives $\delta \mathcal{E}(\cdot, \xi), \delta \mathcal{G}(\cdot, \xi): D \cap Y \rightarrow \mathbb{R}$ are continuous on $\operatorname{int}_{Y}(D \cap Y)$ for any fixed $\xi \in Y$.

Proof. We must show the continuity of $\delta \mathcal{E}(\eta, \xi)$ and $\delta \mathcal{G}(\eta, \xi)$ for $\alpha \in\left[-\alpha_{0} / 2, \alpha_{0} / 2\right]$ and $\beta \in\left[-\beta_{0} / 2, \beta_{0} / 2\right]$ for $\eta=\alpha \eta_{1}+\beta \eta_{2}$. By a result on the continuity of parameter-dependent integrals, cf. Schilling [38, Theorem 11.4], it is sufficient to show the continuity of the integrands in $\alpha, \beta$ and the uniform boundedness of the integrands in $y|D u|$-a. e. on $\Omega$. By looking at the integrands of $\delta \mathcal{E}(\eta, \xi)$ and $\delta \mathcal{G}(\eta, \xi)$, cf. Lemma 3.10, we see that all functions involved are continuous in $\alpha$ and $\beta$ and we have shown the boundedness of the integrands in the proof of Lemma 3.10. Therefore the derivatives $\delta \mathcal{E}(\cdot, \xi), \delta \mathcal{G}(\cdot, \xi): D \cap Y \rightarrow \mathbb{R}$ are continuous as functions of $\alpha$ and $\beta$ and the assertion follows.

Proof of Theorem 3.1. Let $\eta \in D$ with $\mathcal{G}(\eta)=0$. Then, since $u$ is a minimizer of problem (1.6), (1.7), we have for $v(x)=u(x+\eta(x)), v \in B V(\Omega)$

$$
E(u) \leq E(v)
$$

and since the traces of $u$ and $v$ are equal by Lemma 3.8 also

$$
\mathcal{E}(0)=\int_{\Omega} d|D u| \leq \int_{\Omega} d|D v|=\mathcal{E}(\eta),
$$

i. e. $\eta=0$ is a minimizer of $\mathcal{E}$ under the constraint $\mathcal{G}(\eta)=0$ and (i) of Proposition 3.2 is satisfied. 
Lemma 3.5 gives us (ii) and Lemma 3.10, Lemma 3.11 show that (iii) of Proposition 3.2 is satisfied.

Now we show (iv), i. e. we must find a function $\xi_{0} \in C_{0}^{\infty}\left(\Omega, \mathbb{R}^{n}\right)$ such that

$$
\delta \mathcal{G}\left(0, \xi_{0}\right)=-\int_{\Omega}|u| \operatorname{div} \xi_{0} d x \neq 0
$$

since $\mathcal{Q}(y, 0,0)=\operatorname{div} \xi(y)$. By Ambrosio et al. [2, Theorem 3.99] we know that for any $u \in B V(\Omega)$ also $|u| \in B V(\Omega)$, since it is the composition of the Lipschitz continuous function $|\cdot|$ and a BV-function $u$. Moreover, since $G(u)=0$, we have $|u| \neq 0$ on $\Omega$ and therefore $|D| u||(\Omega)>0$. Using the definition of the total variation for $B V$-functions we can conclude that

$$
|D| u||(\Omega)=\sup _{\xi \in C_{0}^{1},\|\xi\|_{\infty} \leq 1} \int_{\Omega}|u| \operatorname{div} \xi d x>0 .
$$

Hence, there exists a function $\xi \in C_{0}^{1}\left(\Omega, \mathbb{R}^{n}\right)$ such that

$$
\int_{\Omega}|u| \operatorname{div} \xi d x>0
$$

By approximation of $\xi$ in the $\|\cdot\|_{\infty}$-norm by a function $\xi_{0} \in C_{0}^{\infty}\left(\Omega, \mathbb{R}^{n}\right)$ we obtain also

$$
\int_{\Omega}|u| \operatorname{div} \xi_{0} d x>0
$$

i. e. (iv) is satisfied.

Thus by applying Proposition 3.2 we get

$$
\delta \mathcal{E}(0, \xi)=\lambda \delta \mathcal{G}(0, \xi)
$$

for all $\xi \in C_{0}^{\infty}\left(\Omega, \mathbb{R}^{n}\right)$ and some $\lambda \in \mathbb{R}$. Since we have

$$
\mathcal{Y}(y, 0,0)^{-1}=\mathcal{Y}(y, 0,0)^{T}=\mathrm{id}
$$

and $\operatorname{det} \mathcal{Y}(y, 0,0)^{-1}=1$, using Lemma 3.10 we get

$$
\int_{\Omega}\langle z, D \xi z\rangle-\operatorname{div} \xi d|D u|=-\lambda \int_{\Omega}|u| \operatorname{div} \xi d x
$$


for all $\xi \in C_{0}^{\infty}\left(\Omega, \mathbb{R}^{n}\right), z$ as in (3.4), and some $\lambda \in \mathbb{R}$.

For a minimizer $u \in B V(\Omega)$ of (1.6), (1.7) we know that $E(u)=h(\Omega)$, cf. (1.20) and that $u$ minimizes the Rayleigh quotient, cf. (1.19). For any other function $v \in B V(\Omega)$ with $v \neq 0$ we have

$$
\frac{E(v)}{G(v)+1} \geq \frac{E(u)}{G(u)+1}=E(u)=h(\Omega),
$$

i. e. $u$ is also a minimizer of the functional

$$
v \mapsto E(v)-h(\Omega)(G(v)+1)
$$

with $E(u)-h(\Omega)(G(u)+1)=0$, cf. also (1.17). Taking $v(x, t)=u(x+t \xi(x))$ for fixed $\xi \in X$ we have $v(\cdot, t) \in B V(\Omega)$ for all $t \in\left(-t_{0}, t_{0}\right)$ and $v$ has the same trace as $u$ on $\partial \Omega$, which does not depend on $t$, cf. Lemma 3.8. Then the functional in (3.43) is equivalent to

$$
\mathcal{E}(t \xi)+\int_{\partial \Omega}|v(x, t)| d \mathcal{H}^{n-1}-h(\Omega)(\mathcal{G}(t \xi)+1)
$$

By calculating the directional derivative of (3.44) in $t=0$ we obtain

$$
\delta \mathcal{E}(0, \xi)-h(\Omega) \delta \mathcal{G}(0, \xi)=0
$$

for each $\xi \in C_{0}^{\infty}\left(\Omega, \mathbb{R}^{n}\right)$ and $\eta \equiv 0$, which corresponds to (3.42) with $\lambda=h(\Omega)$. 


\section{Bibliography}

[1] F. Alter, V. Caselles. Uniqueness of the Cheeger set of a convex body. To appear in Nonlinear Anal.

[2] L. Ambrosio, N. Fusco, D. Pallara. Functions of Bounded Variation and Free Discontinuity Problems. Clarendon Press, Oxford, 2000.

[3] A. Anane, N. Tsouli. On the second eigenvalue of the p-Laplacian. Nonlinear partial differential equations (Fés, 1994) Pitman Res. Notes Math. Ser., 343, Longman, Harlow, 1-9, 1996.

[4] F. Andreu-Vaillo, V. Caselles, J. M. Mazón. Parabolic Quasilinear Equations Minimizing Linear Growth Functionals. Birkhäuser, Basel, 2004.

[5] D. S. Bernstein. Matrix Mathematics. Princeton University Press, Princeton, Oxford, 2005.

[6] K. Borsuk. Theory of Retracts. Polish Scientific Publishers, Warszawa, 1967.

[7] A. Canino, M. Degiovanni. Nonsmooth critical point theory and quasilinear elliptic equations. In: Topological Methods in Differential Equations and Inclusions, 1-50, Montreal, 1994, NATO ASI Series C 472, Dordrecht: Kluwer Academic Publishers, 1995.

[8] A. Canino, U. Perri. Constrained problems in Banach spaces with an application to variational inequalities. Nonlinear Anal. 24, 839-856, 1995.

[9] V. Caselles, A. Chambolle, M. Novaga. Some remarks on uniqueness and regularity of Cheeger sets. Preprint. 
[10] J. Cheeger. A lower bound for the smallest eigenvalue of the Laplacian, in: Problems in Analysis, A Symposium in Honor of Salomon Bochner, Ed.: R.C. Gunning. Princeton Univ. Press, 195-199, 1970.

[11] P. G. Ciarlet. Mathematical Elasticity. Volume 1: Three Dimensional Elasticity. North-Holland, Amsterdam, London, New York, Tokyo, 2004.

[12] F. H. Clarke. Optimization and Nonsmooth Analysis. John Wiley \& Sons, New York, 1983.

[13] O. Cornea, G. Lupton, J. Oprea, D. Tanré. Lusternik-Schnirelmann Category. American Mathematical Society, 2003.

[14] J.-N. Corvellec, M. Degiovanni, M. Marzocchi. Deformation properties for continuous functionals and critical point theory. Topol. Methods Nonlinear Anal. 1, 151-171, 1993.

[15] M. Degiovanni, M. Marzocchi. A Critical Point Theory for Nonsmooth Functionals. Ann. Mat. Pura Appl. (4) 167, 73-100, 1994.

[16] M. Degiovanni, F. Schuricht. Buckling of nonlinearly elastic rods in the presence of obstacles treated by nonsmooth critical point theory. Math. Ann. 311, 675-728, 1998.

[17] F. Demengel. Functions Locally Almost 1-harmonic. Appl. Anal. 83, 865-896, 2004.

[18] J. I. Díaz. Nonlinear partial differential equations and free boundaries, Volume I Elliptic equations. Research Notes in Mathematics 106, Pitman Advanced Publishing Program, Boston, London, Melbourne, 1985.

[19] E. DiBenedetto. $C^{1+\alpha}$ local regularity of weak solutions of degenerate elliptic equations. Nonlinear Anal. 7, 827-850, 1983.

[20] P. Drábek, S. B. Robinson. On the Generalization of the Courant Nodal Domain Theorem. J. Differential Equations 181, 58-71, 2002.

[21] J. Dugundji. An Extension of Tietze's Theorem. Pacific J. Math. 1, 353-367, 1951. 
[22] L. C. Evans, R. F. Gariepy. Measure Theory and Fine Properties of Functions. CRS Press, Boca Raton, 1992.

[23] E. Fadell. The relationship between Ljusternik-Schnirelman category and the concept of genus. Pacific J. Math. 89, 1, 33-42, 1980.

[24] V. Fridman, B. Kawohl. Isoperimetric estimates for the first eigenvalue of the p-Laplace operator and the Cheeger constant. Comment. Math. Univ. Carolin., 44, 659-667, 2003.

[25] J.P. García Azorero, I. Peral Alonso. Existence and nonuniqueness for the p-Laplacian: nonlinear eigenvalues. Comm. Partial Differential Equations, 12, 1389-1430, 1987.

[26] R.F. Gariepy, W.P. Ziemer. A regularity condition at the boundary for solutions of quasilinear elliptic equations. Arch. Ration. Mech. Anal., 67, 25-89, 1977.

[27] M. Giaquinta, S. Hildebrandt. Calculus of Variations I. Springer-Verlag, Berlin, Heidelberg, New York, 1996.

[28] E. Gonzalez, U. Massari, I. Tamanini. Minimal boundaries enclosing a given volume. Manuscripta Math. 34, 381-395, 1981.

[29] B. Kawohl, Th. Lachand-Robert. Characterization of Cheeger sets for convex subsets of the plane. Pacific J. Math. 225, 103-118, 2006.

[30] B. Kawohl, P. Lindqvist. Positive eigenfunctions for the p-Laplace operator revisited. Analysis 26, 539-544, 2006.

[31] B. Kawohl, F. Schuricht. Dirichlet problems for the 1-Laplace operator, including the eigenvalue problem. Commun. Contemp. Math. 9, 515-543, 2007.

[32] P. Lindqvist. On a nonlinear eigenvalue problem. Lecture notes, www.math.helsinki.fi/ analysis/GraduateSchool/lindqvist/nonlin.pdf

[33] A. M. Matei. First eigenvalue for the p-Laplace operator. Nonlinear Anal. 39, 1051-1061, 2000. 
[34] Z. Milbers, F. Schuricht. Existence of a sequence of eigensolutions for the 1-Laplace operator. Preprint MATH-AN-06-2008, TU Dresden.

[35] Z. Milbers, F. Schuricht. Some special aspects related to the 1-Laplace operator. Preprint MATH-AN-04-2008, TU Dresden.

[36] E. Parini. Cheeger sets in the non-convex case. Tesi di laurea, Università degli studi di Milano, 2006.

[37] P. Rabinowitz. Some aspects of nonlinear eigenvalue problems. Rocky Mountain J. Math. 3, 161-202, 1973.

[38] R. Schilling. Measures, Integrals and Martingales. Cambridge University Press, 2005.

[39] E. Stredulinsky, W. P. Ziemer. Area minimizing sets subject to a volume constraint in a convex set. J. Geom. Anal. 7, 653-677, 1997.

[40] E. Zeidler. Nonlinear Functional Analysis and its Applications I: Fixed-Point Theorems. Springer-Verlag, New York, 1985.

[41] E. Zeidler. Nonlinear Functional Analysis and its Applications III: Variational Methods and Optimization. Springer-Verlag, New York, 1985. 


\section{Versicherung}

Hiermit versichere ich, dass ich die vorliegende Arbeit ohne unzulässige Hilfe Dritter und ohne Benutzung anderer als der angegebenen Hilfsmittel angefertigt habe. Die aus fremden Quellen direkt oder indirekt übernommenen Gedanken sind als solche kenntlich gemacht. Die Arbeit wurde bisher weder im Inland noch im Ausland in gleicher oder ähnlicher Form einer anderen Prüfungsbehörde vorgelegt.

Die vorliegende Arbeit wurde am Mathematischen Institut der Universität zu Köln unter der Betreuung von Prof. Dr. F. Schuricht begonnen und am Institut für Analysis der Technischen Universität Dresden, ebenfalls unter der Betreuung von Prof. Dr. F. Schuricht, fertig gestellt.

Ich erkenne die Promotionsordnung der Fakultät Mathematik und Naturwissenschaften der TU Dresden vom 20. März 2000 an.

Dresden, den 12.1.2009 